\title{
Testing and analysis of solid polymers under large monotonic and long-term cyclic
} deformation[Instruction: You have done excellent work. Please, consider our comments below. You have confused the symbols Ivarphi (for free volume in our manuscript, see Fig. 6) and \phi (for angular twist in our manuscript, see p. 7). Please, make the corrections.]

The corrections made in this section will be reviewed and approved by a journal production editor.

Thierry Barriere ${ }^{\mathrm{a}}$, Xavier Gabrion ${ }^{\mathrm{a}}$, Sami Holopainen ${ }^{\mathrm{b}, *}$ sami.holopainen@tuni.fi, Jarno Jokinen ${ }^{\mathrm{c}}$

${ }^{a}$ Univ. Bourgogne Franche-Comté, FEMTO-ST Institute, CNRS/UFC/ENSMM/UTBM, Department of Applied Mechanics, 25000, BESANÇON-FR, France

' Tampere University, Department of Civil Engineering, FI-33014, Tampere, Finland

${ }^{\mathbf{c}}$ Tampere University, Department of Materials Science and Environmental Engineering, FI-33014, Tampere, Finland

*Corresponding author.

\begin{abstract}
The deformation behavior of solid polymers under isothermal, quasi-static loadings is investigated. A comprehensive test program consisting of tension, torsion, and combinations thereof was conducted on a polycarbonate polymer under both monotonic and long-term cyclic loadings. The effects of different loading modes, creep load conditions, mean stress, stress amplitude, and loading rate are addressed. The possibility of simulating costly tests with existing models is also demonstrated. A viscoelastic-viscoplastic constitutive model proposed by Barriere et al. 2019 is applied for this purpose. Compared to state-of-the-art models, this model requires a reduced set of material parameters to be defined. The validation experiments demonstrate the model robustly predicts various loading scenarios. In light of both the experimental and model results, the material shows an apparent hardening with increasing loading rates, and the ratcheting strain increases with the stress amplitude and mean stress. When applying the same stress ratio, stress rate, and maximum axial and torsional stresses relative to the strengths, the superimposed tension increased the torsional ratcheting for all
\end{abstract}


load combinations. The ratcheting-fatigue failure interaction is also investigated. The experimental data show the interaction at least $90 \%$ of the fatigue life, which the important observation is used for the development of a fatigue model.

Keywords: Viscoelasticity-plasticity; Multi-axial plasticity; Simulation of testing; Ratcheting; Fatigue damage

\section{Introduction}

Solid polymers are widely applied in engineering components that must withstand multi-axial cyclic loadings that ultimately leads to fatigue failure or large ratcheting strains, i.e. a cyclic accumulation of inelastic deformation during load-controlled loadings with non-zero mean stress. Representative examples are components in sporting goods, vehicles, and aeronautic equipment. The components may be further strengthened by polymer coatings. Concurrently, the service failures of engineering components due to fatigue have been believed to be the main cause of immense financial losses, Beesley et al. (2017). Consequently, material testing plays a very important role on the design and safety assessment of engineering components. However, such tests take considerable time and affect costs. For this reason, it is worth developing appropriate models to replace resource-intensive tests.

Despite various applications, the volume of research investigating the effects of loading history and multi-axial ratcheting on service life has remained low to date, Lu et al. (2016). The research in this field has concentrated on metallic materials and certain crystalline polymers, as reviewed by Wright et al. (2003); Avanzini (2008); Zhu et al. (2017); Krenk and Tidemann (2017); Qi et al. (2019); Chen et al. (2019). Krairi and Doghri (2014) investigated the interaction between multi-axial deformation (combined tension and torsion) and damage on Nylon 101 semi-crystalline polymer when the number of cycles is low. Beesley et al. (2017) experimentally investigated low-cycle fatigue in a nickel-based alloy and $\mathrm{Lu}$ et al. (2016) short-term ratcheting in a polycarbonate (PC) polymer. Yu et al. (2017) showed that their model for an ultra-high molecular weight polyethylene is accurate under low-cycle loadings. Considering the longer fatigue lives and ratcheting of polymers, the focus has been on uniaxial cyclic loadings, Kim and Lu (2008); Xi et al. (2015); Wang et al. (2016); Kanters et al. (2016); Hughes et al. (2017); Holopainen et al. (2017); Holopainen and Barriere (2018); Barriere et al. (2019); Krairi et al. (2019). The experimentation and models for PC proposed in James et al. (2013); Ravi Chandran (2016) are studied under uniaxial cyclic loadings and are implemented to detect crack growth in tiny zones (not applied at component level) to define long-term fatigue lives.

Chen et al. (2015) investigated the interaction between multi-axial ratcheting (combined tension and torsion) and fatigue in an ABS-polymer, showing that the celebrated Basquin-model (in 1910) for fatigue must be modified. A hyperelastic-viscoplastic model in Shojaei and Volgers (2018) and a parallel rheological network model (elastic-viscoplastic) in Qi et al. (2019) provide capable tools for the investigation of both low- and high-cycle regimes in highly crystalline and semi-crystalline polymers. Considering especially glassy polymers, however, both the viscoelastic and plastic elements are needed to accurately predict the long-term creep/recovery and thus the long-term cyclic deformation behavior (ratcheting and shape/area of the loops). Only the models introduced in Anand and Ames (2006); Janssen et al. (2008); Jiang et al. (2015); Krairi et al. (2019); Barriere et al. (2019) include these elements, despite being solely demonstrated under one-dimensional loadings.

It can be concluded that the research on polymers under multi-axial and long-term cyclic loadings is very limited. The present research focuses on this topic and concerns all the elements including experimentation, modeling, and simulation of testing. Lu et al. (2016) introduced an experimental data set (beyond standards) of PC for 
multi-axial short-term ratcheting, including various combinations of tension and torsion (linear, butterfly-types, squared, and rhombic). Our research augments their work (based on the standards) by investigating shear ratcheting strain, phase difference, and long-term cyclic deformation behavior including fatigue life. Another endeavor of this work is to demonstrate that the current, most capable models for polymers begin to show their worth when simulating multi-axial tests. The applied model, which is capable of predicting both extremely large deformations and cyclic deformation behaviour, was introduced in Barriere et al. (2019). This model is based on the renowned model for glassy polymers proposed by Haward and Thackray (1968), which model is augmented by a few thermodynamically relevant internal state variables that enable the prediction of the cyclic deformation behavior in three dimension. Compared with capable state-of-the-art models, Anand and Ames (2006); Jiang et al. (2015), the model has several advantages. For example, the number of material parameters is reduced, ( Barriere et al., 2019, Introduction). In this work, capability of the model is investigated under multi-axial deformations (combined tension and torsion), and the model is augmented with a fatigue model for investigating service lives.

The article constitutes of the following parts:

1. Experimentation including

(a) tests for monotonic and cyclic tension and torsion,

(b) tests for combined multi-axial cyclic tension and torsion;

2. Kinematics including details for the tests;

3. A brief summary of the constitutive model;

4. Description of the proposed fatigue model;

5. Test and model results, their comparison, and simulation of testing.

The effects of loading path, creep load conditions, mean stress, stress amplitude, and loading rate on the deformation behavior of a commonly used PC polymer is addressed in light of both experimental and model results. Also, ratcheting deformation-fatigue failure interaction is investigated. The article closes with conclusions and needs for future research.

\section{Experimentation}

\subsection{Manufacture of specimens}

The PC polymer (Lexan ${ }^{\circledR}$ 223R granulate) applied in the tests is suitable for cyclic loading conditions, as it is characterized by high impact and fatigue resistances. The geometry of the injection-molded tensile specimen illustrated in Fig. 1 is in accordance with the standard (type IV specimen), ASTM D638 (2003); Barriere et al. (2018). The only deviation from the standard is that the gauge length $L_{\mathrm{g}}$ was chosen to be $40 \mathrm{~mm}$ to obtain better compatibility with the extensometer, with its gauge length of $25 \mathrm{~mm}$; see Table 1 . The geometry of the torsional test specimen according to the standard ASTM E2207 (2002) is shown in Fig. 2. This specimen with the lower inner diameter of $9 \mathrm{~mm}$ was designed to avoid premature buckling under cyclic torsion.

alt-text: Fig. 1

Fig. 1 


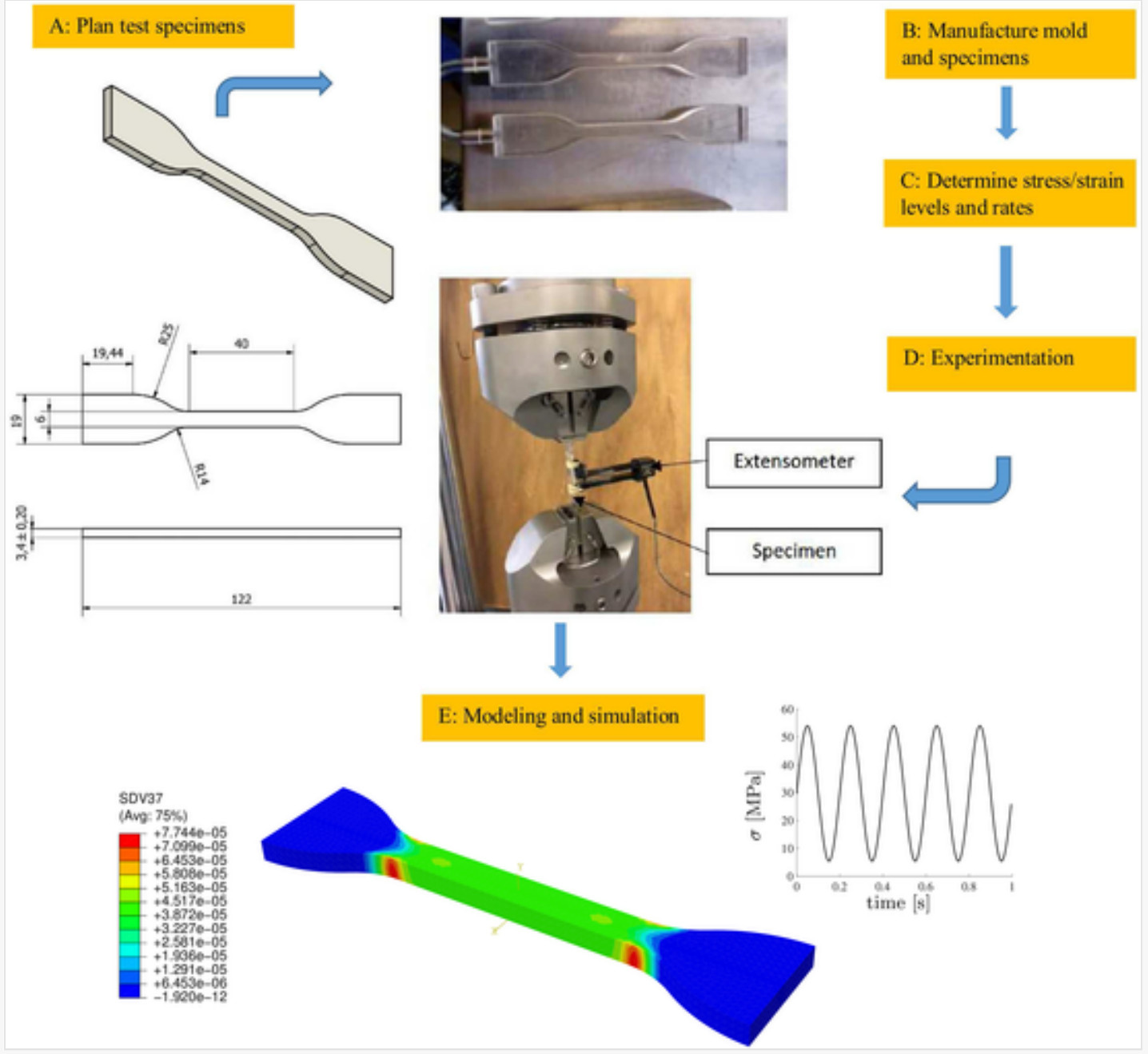

Flowchart of the test program consisting of steps A-B-C-D-E. Geometry of the tensile test specimens (mm), tensile test setup, and a simulation result for free volume after 60 cycles.

alt-text: Table 1

Table 1

(i) The table layout displayed in this section is not how it will appear in the final version. The representation below is solely purposed for providing corrections to the table. To preview the actual presentation of the table, please view the Proof.

Details for the testing arrangements.

\begin{tabular}{|l|l|l|l|l|}
\hline Test & ASTM-standard & Extensometer & Capacity of extensometer & Data acquisition (Hz) \\
\hline Monotonic tension & $\underline{\text { D638-03 }}$ & Instron 2620.601 & 5 mm, i.e. 20\% strain & 20 \\
\hline Creep and recovery & $\underline{\text { D2990-01 }}$ & Instron 2620.601 & 5 mm, i.e. $20 \%$ strain & 1 \\
\hline Cyclic tension & D2990-01 & Instron 2620.601 & 5 mm, i.e. $20 \%$ strain & 1000 \\
\hline Monotonic torsion & $\underline{E 2207}$ & MTS 632.68F & $12^{\circ}$, i.e. $3 \%$ shear strain & 15 \\
\hline Cyclic torsion & $\underline{E 2207}$ & MTS 632.68F & $12^{\circ}$, i.e. $3 \%$ shear strain & 200 \\
\hline Cyclic tension-torsion & E2207 & MTS 632.68F & 2.5 mm, i.e. $10 \%$ strain; & $100,10^{\dagger \dagger}$ \\
\hline
\end{tabular}


$\dagger$ Not applied to the loading.

$\dagger 100 \mathrm{~Hz}$ in linear and elliptic tests, otherwise $10 \mathrm{~Hz}$.

alt-text: Fig. 2

Fig. 2

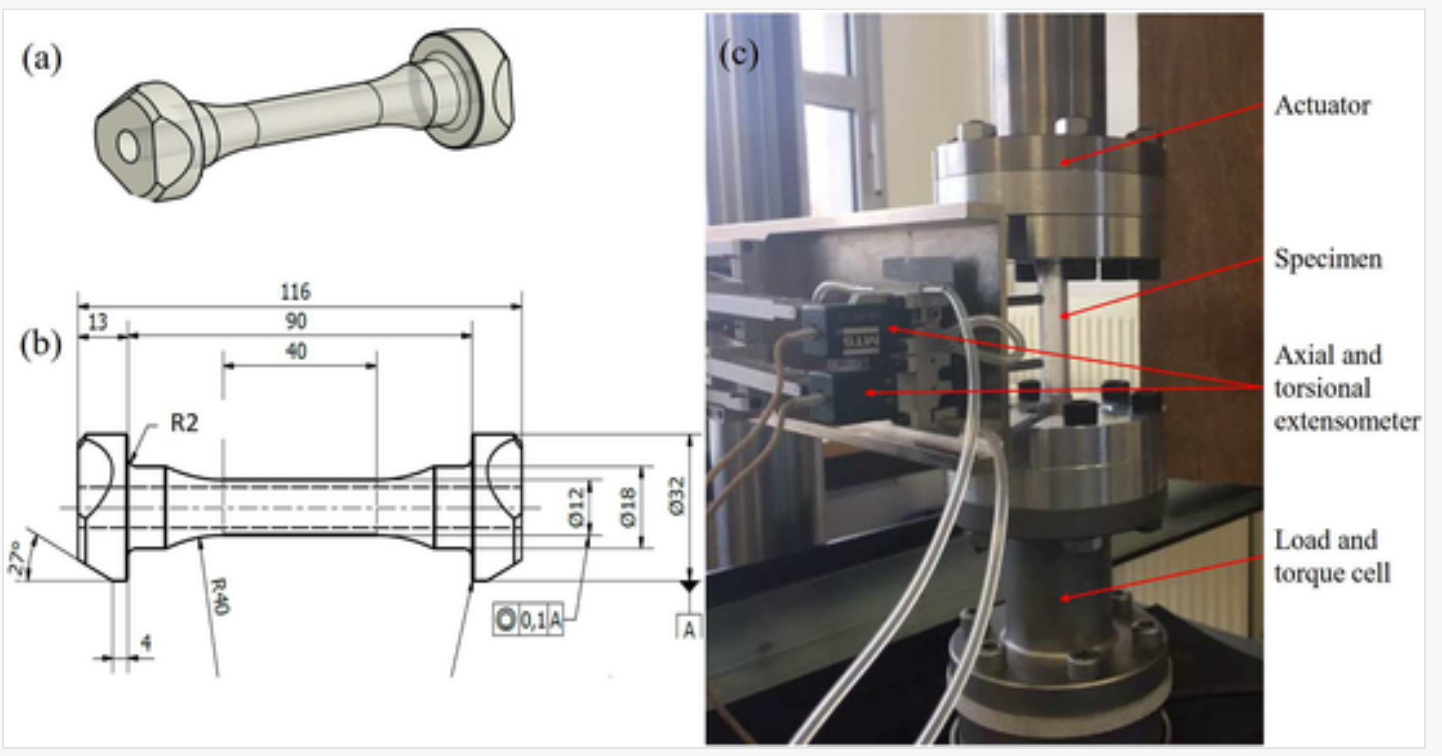

Design of the torsion specimen (a), dimension of the specimen (mm) (b), and setup for the tests (c).

Because the tests are sensitive to defects of the specimens, particular attention was paid to their quality. For instance, the defects of injected torsion specimens were analyzed with Werth video-check and X-ray tomography. Moreover, the shape was analyzed by using optical 3D metrology (Alicona analyser). The observed maximum surface fault was $0.03 \mathrm{~mm}$. The influence of this fault on the test results can be considered to be negligible, because the errors in the outer radius $(6 \mathrm{~mm})$ and cross-section of the gauge section are only $0.5 \%$ and $1.1 \%$, respectively. Each test was conducted at least twice. Of these results, the best-fit result was selected for the presentation herein.

\subsection{Uniaxial tensile tests}

The tests were performed using an Instron ${ }^{\circledR}$ Electropulse E10000 machine with a load capacity of $10 \mathrm{kN}$ and a maximum displacement of $\pm 30 \mathrm{~mm}$ :

- Displacement-controlled monotonic tests at different strain rates $\dot{\varepsilon}=0.001 \mathrm{~s}^{-1}, \dot{\varepsilon}=0.01 \mathrm{~s}^{-1}$, and $\dot{\varepsilon}=0.1 \mathrm{~s}^{-1}$ [Instruction: Space here]until rupture;

- Repeated creep and recovery for a total of $5 \mathrm{~h}$;

- Force controlled cyclic tests at $R=0.1$ and $R=0.5$ ( $R$ is the ratio between the minimum and maximum stress) until rupture as $f=5 \mathrm{~Hz}$ (sinusoidal). The values of the maximum stresses were $97 \%, 90 \%, 75 \%, 50 \%$, and $37.5 \%$ of the peak yield stress, $60 \mathrm{MPa} .{ }^{1}$

The first test set is used to investigate the loading response, tensile yield strength, the yield peak stress, and subsequent softening at large strains. The second, isochronous load-controlled test is aimed at investigating the viscous deformation behavior. The last test set is used to investigate the influence of stress amplitude and mean 
stress on the cyclic deformation behavior (unloading response and ratcheting) and fatigue life. The final maximum stress was reached gradually within the first twenty cycles.

The axial elongation $u$ with respect to the force $F$ was recorded by the machine. The elongation was also measured by an extensometer, see Table 1 and the results of the extensometer and machine were essentially the same. The capacity of the extensometer was sufficient for all the tests until rupture. The strain was defined by $\varepsilon:=u / L$, where $L$ is the gauge length of the extensometer used. Then, the stress was defined by $\sigma:=F / A$, where $A$ is the initial cross-sectional area of the specimen. ${ }^{2}$

\subsection{Torsional tests}

The same testing machine as that used for tension was used for the torsional tests, with a load cell capacity of $\pm 100 \mathrm{Nm}$ and an angle of $135^{\circ}$. Although the standard applied was intended for metals, see Table 1 , the dimension of the specimen and practices were easily variable for polymers with the employed testing machine. The following tests under force control were realized:

- Monotonic torsional tests where the torque speed is $1 \mathrm{Nms}^{-1}$ [Instruction: space here]until rupture;

- Progressively repeated loading (PRL) tests where the torque speed is $1 \mathrm{Nms}^{-1}$ and data acquisition frequency is $75 \mathrm{~Hz}$, see Fig. 3 for the loading path;

- Cyclic tests at $R=0.1$ and $f=1 \mathrm{~Hz}$ (up to rupture) where the values of maximum shear stresses were $90 \%, 82 \%, 75 \%, 65 \%, 50 \%$, and $30 \%$ of the ultimate shear strength, $40 \mathrm{MPa}$.

\section{alt-text: Fig. 3}

\section{Fig. 3}
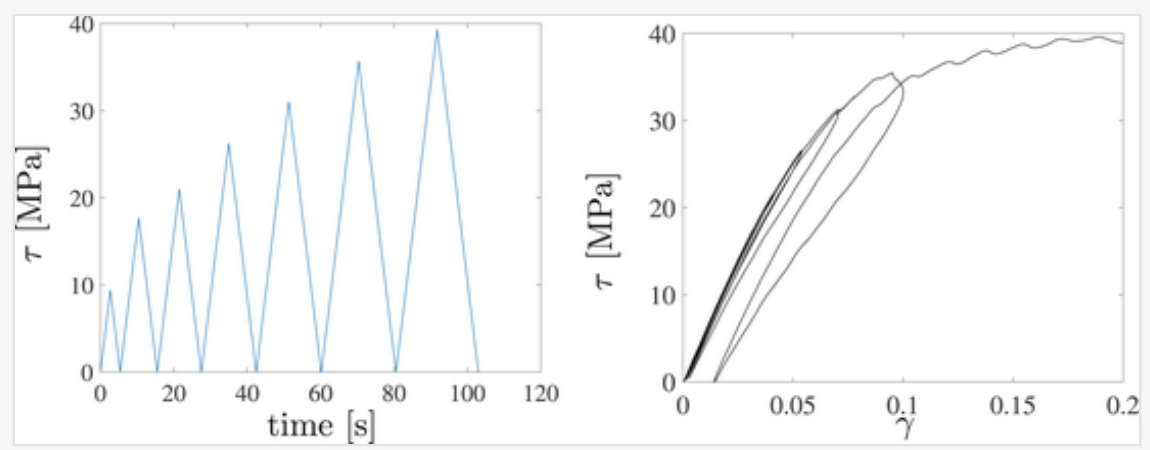

Loading path used in the PRL-tests (left) and observed stress vs strain curve (right). The shear stress response is calculated by (1).

The PRL-tests enable the evaluation of the ultimate shear strength $\left(\tau_{\mathrm{u}}=40 \mathrm{MPa}\right)$ and the shear yield strength, which is approximately $30 \%$ of $\tau_{\mathrm{u}}$ for the PC polymer under study.

The torsion angle was also measured using the tensile/torsional extensometer introduced in Table 1. The range of the extensometer used was sufficient to measure the shear modulus, i.e. the relation between the initial shear strain and stress. To avoid slipping, the extensometer was glued onto the specimen's surface. The shear strain and shear stress are calculated as follows: 
$\gamma=\frac{d_{\mathrm{o}}}{2} \frac{\theta}{L_{\mathrm{g}}}, \quad \tau=\frac{16 T}{\pi\left(d_{\mathrm{o}}^{2}-d_{\mathrm{i}}^{2}\right)\left(d_{\mathrm{o}}+d_{\mathrm{i}}\right)}$

where $d_{\mathrm{o}}$ and $d_{\mathrm{i}}$ are the outer and inner diameters, $\theta$ is the angle in radians (measured by both the machine and extensometer), $L_{\mathrm{g}}$ is the gauge length of the specimen, and $T$ denotes the torque. In the tests, the values were $d_{\mathrm{o}}=12 \mathrm{~mm}, d_{\mathrm{i}}=9 \mathrm{~mm}$, and $L_{\mathrm{g}}=40 \mathrm{~mm}$.

Table 2 displays the mechanical properties extracted from the tests. Based on the standard deviation (SD), the final shear modulus $G=\tau / \gamma$ is approximately $590 \pm 40 \mathrm{MPa}$. It can be observed that the difference between the moduli calculated using the extensometer and machine angles is very small. The observed strain corresponding to the ultimate shear strength of about $40 \mathrm{MPa}$ is nearly 18\%. Additionally, the PRL-tests show a similar value, see Fig. 3. Moreover, as shown in Fig. 3 and Table 2 for the PRL-tests, the residual shear strain after unloading to zero stress is clearly visible, starting from the penultimate cycle.

\section{alt-text: Table 2}

Table 2

Mechanical properties of PC obtained from the monotonic tests and PRL-tests.

\begin{tabular}{|c|c|c|c|c|c|c|}
\hline Units & $\begin{array}{l}\text { Modulus }(0-1.5 \% \text { of } \\
\text { strain) extensometer }\end{array}$ & $\begin{array}{l}\text { Modulus (0-1.5\% } \\
\text { of strain) machine }\end{array}$ & $\begin{array}{l}\text { Strain at } \\
\text { maximum } \\
\text { stress }\end{array}$ & $\begin{array}{l}\text { Maximum } \\
\text { stress }\end{array}$ & $\begin{array}{l}\text { Residual } \\
\text { strain }(\%) \text { at } \\
5 \text { th cycle }\end{array}$ & $\begin{array}{l}\text { Residual } \\
\text { strain }(\%) \text { at } \\
\text { 6th cycle }\end{array}$ \\
\hline & MPa & MPa & $\%$ & MPa & $\%$ & $\%$ \\
\hline $\begin{array}{l}\text { Specimen } \\
1\end{array}$ & 648 & 632 & 16.6 & 39.1 & - & - \\
\hline $\begin{array}{l}\text { Specimen } \\
2\end{array}$ & 629 & 623 & 18.2 & 40.4 & - & - \\
\hline $\begin{array}{l}\text { Specimen } \\
3\end{array}$ & 562 & 576 & 19.4 & 39.4 & - & - \\
\hline $\begin{array}{l}\text { Specimen } \\
1 \mathrm{PRL}\end{array}$ & 535 & 534 & - & - & 0.14 & 1.75 \\
\hline $\begin{array}{l}\text { Specimen } \\
2 \text { PRL }\end{array}$ & 585 & 582 & - & - & 0.09 & 1.24 \\
\hline $\begin{array}{l}\text { Specimen } \\
3 \text { PRL }\end{array}$ & 588 & 582 & - & - & 0.10 & 1.37 \\
\hline Mean & 591 & 588 & 18.1 & 39.6 & 0.10 & 1.50 \\
\hline SD & 38 & 32 & 1.1 & 0.6 & 0.0 & 0.30 \\
\hline $\mathrm{CoV}$ & 6 & 5 & 6.3 & 1.4 & 21.2 & 17.1 \\
\hline
\end{tabular}


From the uniaxial tests, using the initial elastic response (strain $\varepsilon=0.02$ and stress $\sigma=48 \mathrm{MPa}$ ), the elastic modulus is $E=2400 \mathrm{MPa}$ and the shear modulus becomes $G=E /(2(1+\nu))=876 \mathrm{MPa}(\nu=0.37$ being typical for the polymer under study, Holopainen and Barriere (2018)). It can be concluded that the shear modulus obtained from the torsional tests, $590 \pm 40 \mathrm{MPa}$, is considerably lower that observed from the tensional tests. To this end, the value of the elastic modulus was reduced to $E=2100 \mathrm{MPa}$ to capture the initial elastic response more accurate with the model, see (Barriere et al., 2019, Sec. 3.2).

\subsection{Tests for combined tension - torsion}

Tensile and torsional tests were benefited in selecting suitable stress levels for multi-axial ratcheting tests, i.e. the maximum stress values for tension and torsion were well below the observed peak yield stresses to avoid premature failure of the specimens. The torque, axial force, axial elongation, and torsion angle were measured by the machine. The axial elongation and the torsion angle were also measured by the extensometer. The detailed load-controlled loading paths are shown in Fig. 4. The different loading paths are termed: linear, elliptic, butterfly-type, squared, and rhombic. For the linear and elliptic loadings, the frequency is $1 \mathrm{~Hz}$. For other tests, the cycles have a duration of 20 s, i.e. the frequency is $0.05 \mathrm{~Hz}$.

\section{alt-text: Fig. 4}

Fig. 4
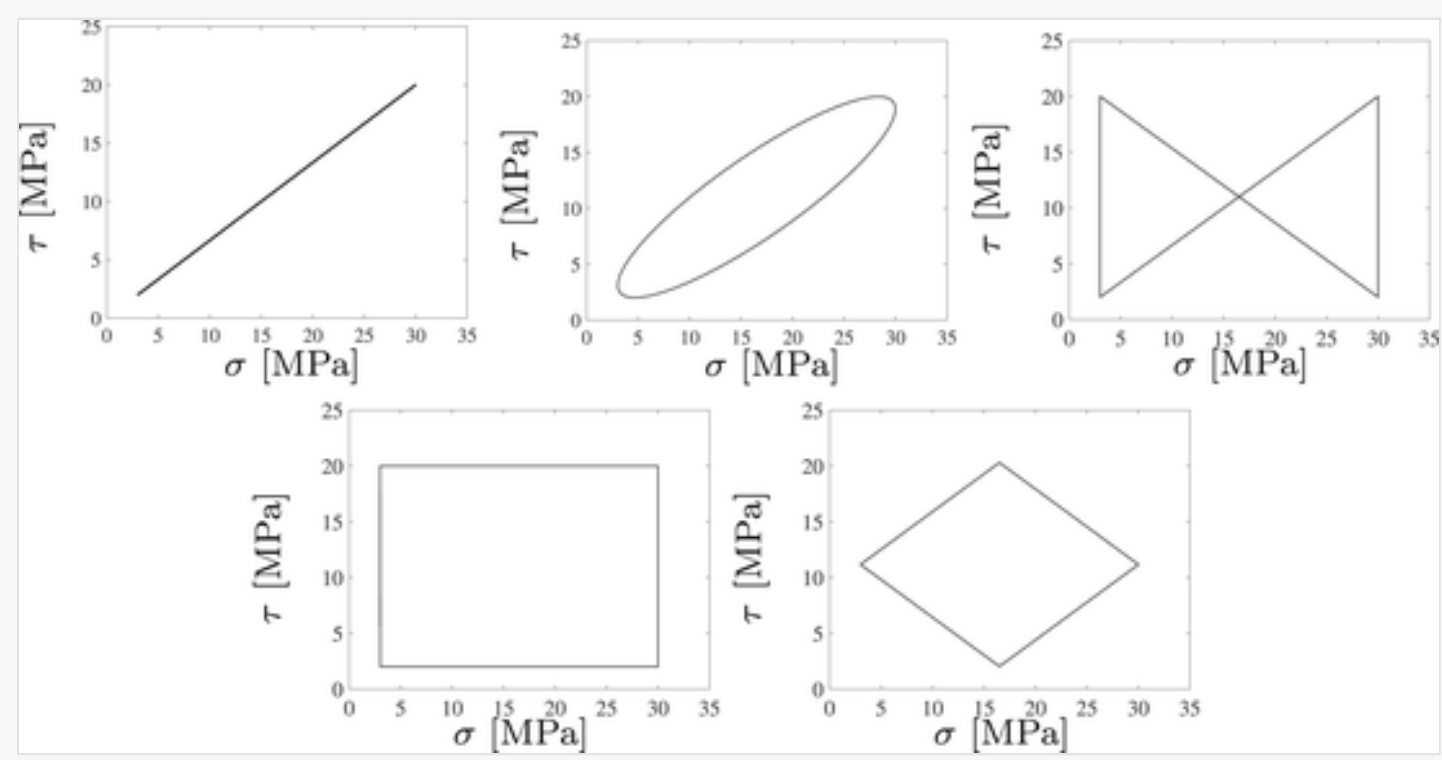

Loading paths applied under load-controlled multi-axial tests. The symbols $\sigma$ and $\tau$ mean the axial and shear stresses (Cauchy), respectively.

\section{Kinematics}

Large deformations under both monotonic and cyclic ratcheting loadings are discussed. The applied model is founded on the Kröner-Lee decomposition, i.e.

$$
\boldsymbol{F}=\boldsymbol{F}^{\mathrm{e}} \boldsymbol{F}^{\mathrm{vep}}
$$

where $\boldsymbol{F}^{\mathrm{e}}$ and $\boldsymbol{F}^{\text {vep }}$ define the local deformation for the intermediate placement $\overline{\boldsymbol{N}}$ due to the elastic and viscoelastic-plastic mechanisms of the chain network, respectively. The symmetric and positive definite stretch 
tensor $\boldsymbol{v}^{\mathrm{e}}$ is defined by the polar decomposition

$$
F^{\mathrm{e}}=v^{\mathrm{e}} \boldsymbol{R}^{\mathrm{e}}
$$

wherein $\boldsymbol{R}^{\mathrm{e}}$ is the rotation associated with elastic deformation. ${ }^{3}$ Purely elastic, reversible deformation is given in terms of a symmetric spatial stretch tensor,

$$
\boldsymbol{b}^{\mathrm{e}}:=\boldsymbol{F}^{\mathrm{e}} \boldsymbol{F}^{\mathrm{e}, \mathrm{T}}=:\left(\boldsymbol{v}^{\mathrm{e}}\right)^{2}
$$

where the tensor transpose is symbolized by $\mathrm{T}$. The viscoelastic-plastic deformation in the stress-free intermediate placement is governed by

$$
\overline{\boldsymbol{C}}^{\mathrm{vep}}:=\boldsymbol{F}^{\mathrm{vep}} \boldsymbol{F}^{\mathrm{vep}, \mathrm{T}}
$$

The quantities in this intermediate placement are accentuated by the bar. Their counterparts in the current placement are obtained by the push-forward operation $\varphi_{*}(\cdot):=\boldsymbol{F}^{\mathrm{e}-\mathrm{T}}(\cdot) \boldsymbol{F}^{\mathrm{e}-1}$. Furthermore, the symmetric stretch-like tensors $\overline{\boldsymbol{C}}^{\mathrm{vp}}$ and $\overline{\boldsymbol{C}}^{\mathrm{ve}}$ are used to characterize the internal state and its change due to the viscoplastic and viscoelastic deformations: elements (1) and (2) in Fig. 6. More detailed account including the rate kinematics of the model is given in Barriere et al. (2019).

alt-text: Fig. 5

Fig. 5
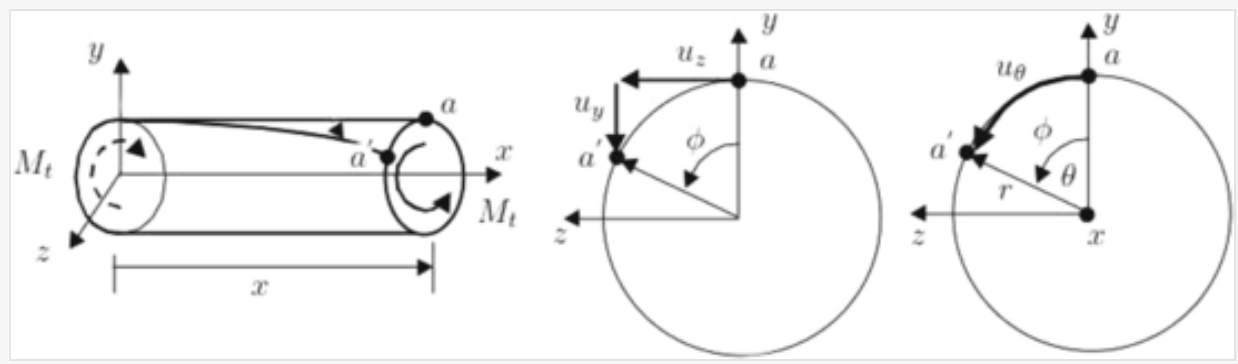

Kinematics of a circular cylinder under torque $M_{t}$.

alt-text: Fig. 6

Fig. 6 
Based on the deformation in (6), i.e. $\boldsymbol{X}=(X, \bar{R} \Theta, R)$ and $\boldsymbol{x}=\left(x, \bar{R} \lambda_{\mathrm{R}} \theta, r\right)$, the matrix form of the deformation gradient becomes

$$
\boldsymbol{F}=\frac{\partial \boldsymbol{x}}{\partial \boldsymbol{X}}=\left[\begin{array}{ccc}
\lambda & 0 & 0 \\
\gamma & \lambda_{\mathrm{R}} & 0 \\
0 & 0 & \lambda_{\mathrm{R}}
\end{array}\right]
$$

where $\gamma:=\lambda \varphi r$ [Instruction: use $\backslash$ phi, not $\mid$ varphi]. Under pure torsion, $\lambda=\lambda_{\mathrm{R}}=1$, and under uniaxial tension, $\varphi=0$ [Instruction: use \phi, not lvarphi]when the component matrix is diagonal.

Under combined tension and torsion, the form of $\boldsymbol{F}^{\mathrm{e}}$ and $\boldsymbol{F}^{\mathrm{vep}}$ is the same as in (7) for the total deformation. Let $\lambda^{\text {vep }}$ and $\gamma^{\text {vep }}$ be the viscoelastic-plastic axial stretch and shear strain, respectively. Regarding viscoelasticplastic deformation as isochoric, i.e. $\operatorname{det}\left(\boldsymbol{F}^{\text {vep }}\right)=1$, yields

$$
\boldsymbol{F}^{\mathrm{vep}}=\left[\begin{array}{ccc}
\lambda^{\text {vep }} & 0 & 0 \\
\gamma^{\text {vep }} & 1 / \sqrt{\lambda^{\text {vep }}} & 0 \\
0 & 0 & 1 / \sqrt{\lambda^{\text {vep }}}
\end{array}\right]
$$

Let then $\lambda^{\mathrm{e}}$ and $\gamma^{\mathrm{e}}$ be the elastic axial stretch and shear strain, respectively. Following the celebrated Boyce et al. (1989) model, the logarithmic stretch $\ln \boldsymbol{v}^{\mathrm{e}}$ is used in the elastic constitutive description. Because the plane stress state prevails in the thin-walled specimen, the only non-zero stress components are $\sigma_{\mathrm{x}}:=\sigma$ and $\tau_{\mathrm{x} \theta}:=\tau$. Based on the elastic constitutive description $\ln \lambda^{\mathrm{e}}=\sigma / E$, where $E$ is the Young's modulus. The shear strain is defined by $\gamma^{\mathrm{e}}=\tau / G$, where $G$ is the shear modulus. Taking the Poisson effect into account, the component matrix takes the form

$$
\ln \left(v^{\mathrm{e}}\right)=\left[\begin{array}{ccc}
\ln \lambda^{\mathrm{e}} & \gamma^{\mathrm{e}} & 0 \\
\gamma^{\mathrm{e}} & -v \ln \lambda^{\mathrm{e}} & 0 \\
0 & 0 & -v \ln \lambda^{\mathrm{e}}
\end{array}\right]
$$

where the matrix logarithm is applied. When using displacement-control, the total deformation gradient $\boldsymbol{F}$ by (7) is known. Once $\boldsymbol{F}^{\mathrm{e}}$ has been solved numerically, $\boldsymbol{F}^{\text {vep }}$ can be obtained from the decomposition (2). Under force control, by contrast, the elastic deformation according to (9) is known based on the constitutive law. However, the rotation $\boldsymbol{R}^{\text {e }}$ remains undetermined under both control methods, which renders the stress-free intermediate placement undetermined. This issue is discussed in the Appendix.

\section{Constitutive theory}

It is necessary to be able to predict loading and unloading responses (shape of the loops), yield stress and strain, softening, anisotropic hardening at very large strains, creep, recovery, and cyclic hardening (ratcheting). The applied model relies on the distinguished model by Haward and Thackray (1968), which is illustrated in Fig. 6. This model includes an elastic spring a) and a nonlinear spring c), which result in an initial elastic response and an anisotropic effect of material under large deformations. To predict increasing macro-yielding followed by a 
notable softening effect, the model also includes a nonlinear dashpot (1). To improve the predictions of the cyclic hysteresis loops, the model is augmented with a system for viscoelastic micro-mechanisms consisting of a KelvinVoigt-like element (2) arranged in series with the dashpot (1). In addition to the dashpot (1), element (2) governs creep and it is also used to predict recovery; its action together with spring a) is used to model stress relaxation. The model is detailed in Barriere et al. (2019). The numerical solution of the model is conferred in the Appendix.

The model parameters are given in Tables 3 and 4. Most of the model parameters were extracted from a displacement-controlled, monotonic cold drawing test. To refine the predictions of unloading responses, a single load-controlled, uniaxial cyclic test (maximum stress $90 \%$ of the peak yield stress) was also utilized. The first and last hysteresis loops of the cyclic test were used to determine the total elongation or ratcheting strain (parameters $m_{0}$ and $\left.\phi_{\mathrm{cv}}\right)$ [Instruction: use Ivarphi here for free volume, not \phi], see Barriere et al. (2019) for the details on model calibration. In contrast to the closest state-of-the-art models, Anand and Ames (2006); Jiang et al. (2015), which are also able to predict cyclic deformation behavior well (with 32 and 38 material parameters, respectively), only 19 parameters are needed in the present model.

alt-text: Table 3

Table 3

Elastic and viscoelastic model parameters for a PC.

\begin{tabular}{|c|c|c|c|c|c|c|c|c|c|}
\hline Parameter & $E$ & $\nu$ & $\dot{v}_{0}$ & $\alpha$ & $m_{1}$ & $s^{(2)}$ & $c_{1} \cdot 10^{-6}$ & $\mu_{1, \mathrm{sat}}$ & $\mu_{1}^{0}$ \\
\hline Unit .......... & $\mathrm{MPa}$ & & $s^{-1}$ & & & $\mathrm{MPa}$ & $\mathrm{MPa}$ & $\mathrm{MPa}$ & $\mathrm{MPa}$ \\
\hline Value .......... & 2100 & 0.37 & 0.031 & 0.204 & 0.19 & 12 & 4.5 & 2500 & 8000 \\
\hline
\end{tabular}

alt-text: Table 4

Table 4

The table layout displayed in this section is not how it will appear in the final version. The representation below is solely purposed for providing corrections to the table. To preview the actual presentation of the table, please view the Proof.

Viscoplastic parameters $(\phi(0)=0)$.

\begin{tabular}{|c|c|c|c|c|c|c|c|c|c|}
\hline Parameter & $s_{0}$ & $m_{0}$ & $C_{\mathbf{R}}$ & $N$ & $h_{0}$ & $b$ & $g_{0}$ & $s_{\text {cv }}$ & $\phi_{\text {cv }}[$ Instruction: \varphi, not $\backslash$ phi] \\
\hline Unit .......... & $\mathrm{MPa}$ & & $\mathrm{MPa}$ & & $\mathrm{MPa}$ & & & $\mathrm{MPa}$ & \\
\hline Value .......... & 28.0 & 0.037 & 14.0 & 1.65 & 3500 & 600 & 0.015 & 26.5 & 0.0013 \\
\hline
\end{tabular}




\subsection{Prediction of fatigue life}

The proposed continuum based model for fatigue is an extension of the Ottosen et al. (2008) model for highcycle fatigue (HCF) and is based on the concept of an evolving damage variable and an endurance surface moving in stress space. Prerequisite is the existence of an asymptotical extreme of lifetime, i.e. the endurance limit, and fatigue under this limit is suppressed. Contrast to cycle-counting approaches, movement of the endurance surface and damage evolution are defined in terms of stress increments, not of stress cycles. The fatigue model is given in terms of the Cauchy stress $\sigma=1 / J \tau$, while $\tau$ (not bolded) means the shear stress component. The endurance function (endurance surface when $\beta=0$ ) is described by

$$
\beta=\frac{1}{\sigma_{0}}\left(\bar{\sigma}+g_{\mathrm{I}}\left(I_{1} ; a, c\right)+g_{\mathrm{II}}\left(I_{1}, I_{2} ; Q, Q_{b}\right)-\sigma_{0}\right),
$$

where $g_{\mathrm{I}}\left(I_{1} ; a, c\right)=a\left(1-c I_{1}\right) I_{1}, g_{\mathrm{II}}\left(I_{1}, I_{2} ; Q, Q_{b}\right)=\left(Q-Q_{b} \bar{I}_{1}\right) I_{2}, \bar{I}_{1}=\sqrt{I_{1}^{2}}$, and $I_{1}=\operatorname{tr} \sigma:=\sigma: \boldsymbol{i}$ and $I_{2}=1 / 2((\operatorname{tr}$ $\left.\sigma)^{2}-\sigma: \sigma\right)$ are the invariants. Moreover, $\sigma_{0}, a, c, Q$, and $Q_{b}$ are material parameters and

$$
\bar{\sigma}=\sqrt{\frac{3}{2}\left(\sigma^{\operatorname{dev}}-\alpha\right): \mathbb{M}:\left(\sigma^{\operatorname{dev}}-\alpha\right)},
$$

where the coefficient matrix $\mathbb{M}$ is defined as

$$
\left(\boldsymbol{\sigma}^{\mathrm{dev}}-\boldsymbol{\alpha}\right): \mathbb{M}:\left(\boldsymbol{\sigma}^{\mathrm{dev}}-\boldsymbol{\alpha}\right)=\left(\boldsymbol{\sigma}^{\mathrm{dev}}-\boldsymbol{\alpha}\right)_{i i}^{2}+M\left(\boldsymbol{\sigma}^{\mathrm{dev}}-\boldsymbol{\alpha}\right)_{i j}^{2}
$$

$i \neq j=1,2,3 ; M$ is a material parameter. The first invariant $I_{1}$ in the $\beta$ function takes into account the effect of hydrostatic and mean stresses. The coefficient matrix $\mathbb{M}$ allows to adjust the relation between the normal and torsional stresses, and it defines the initial slope of $\beta$. This is demonstrated in Fig. 7(left) as $\sigma=0$. In the original model by Ottosen et al. (2008), only low uniaxial stress states are investigated and $\mathbb{M}$ is considered identity therein. Moreover, the linear relation $A I_{1}$ in the Ottosen et al. (2008) is replaced by the nonlinear functions $g_{\mathrm{I}}$ and $g_{\mathrm{II}}$ to affect a reduction in $\beta$ in heavy normal and shear stresses, respectively. This feature is in line with the experiments. Function $g_{\text {I }}$ also encompasses a large range of mean stresses as demonstrated in Fig. 7(right). ${ }^{4}$

alt-text: Fig. 7

Fig. 7
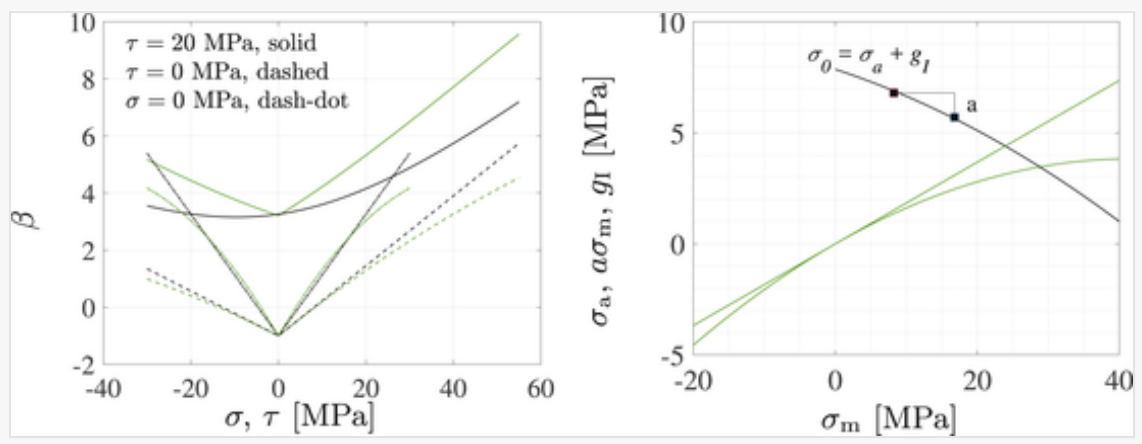
Endurance functions according to (10) (green) and Ottosen et al. (2008) (black) when $\alpha$ is yet small in relation to the stress (left). Functions $A \sigma_{\mathrm{m}}, g_{\mathrm{I}}$ (green), and the Haigh-diagram (black) for 60,000 cycles (right). Markers $\boldsymbol{\Xi}$ are the data points used in the model fitting. (For interpretation of the references to color in this figure legend, the reader is referred to the Web version of this article.)

The backstress quantity $\alpha$ defines the center of the endurance surface, i.e. its evolution is defined by

$$
\dot{\alpha}=C\left(\tau^{\mathrm{dev}}-\alpha\right) \dot{\beta}
$$

where $C$ is a non-dimensional parameter. Fatigue damage only evolves outside the endurance surface, i.e. $\beta \geq 0$. Also, damage never decreases $(0 \leq D \leq 1)$, and it is postulated that damage increases only when $\dot{\beta} \geq 0$. Then, the damage evolution is governed by the exponential form

$$
\dot{D}=\frac{K}{(1-B D)} \exp \left(f\left(\beta ; L_{1}, L_{2}, \vartheta\right)\right) \dot{\beta} \geq 0
$$

where

$$
f\left(\beta ; L_{1}, L_{2}, \vartheta\right):=\left[L_{1}-L_{2}\left(1+\frac{L_{2}}{\vartheta \beta}\left(\exp \left(-\vartheta \frac{\beta}{L_{2}}\right)-1\right)\right)\right] \beta
$$

and $B, K, L_{1}, L_{2}$, and $\vartheta$ are positive parameters. The proposed damage evolution differs from the Ottosen et al. (2008) model for HCF in that the function $f$ for LCF is applied: the function $f$ has the asymptote $L_{1} \beta$ as $\beta$ is near zero in the HCF-regime and $\left(L_{1}-L_{2}\right) \beta$ as $\beta$ is large in the LCF-regime. Parameter $\vartheta$ defines the curvature of how rapidly the second asymptote is achieved, see Fig. 8(left). Moreover, the denominator $(1-B D)$ is applied to provide an expected, increasing damage rate, Junior et al. (2011).

alt-text: Fig. 8

Fig. 8

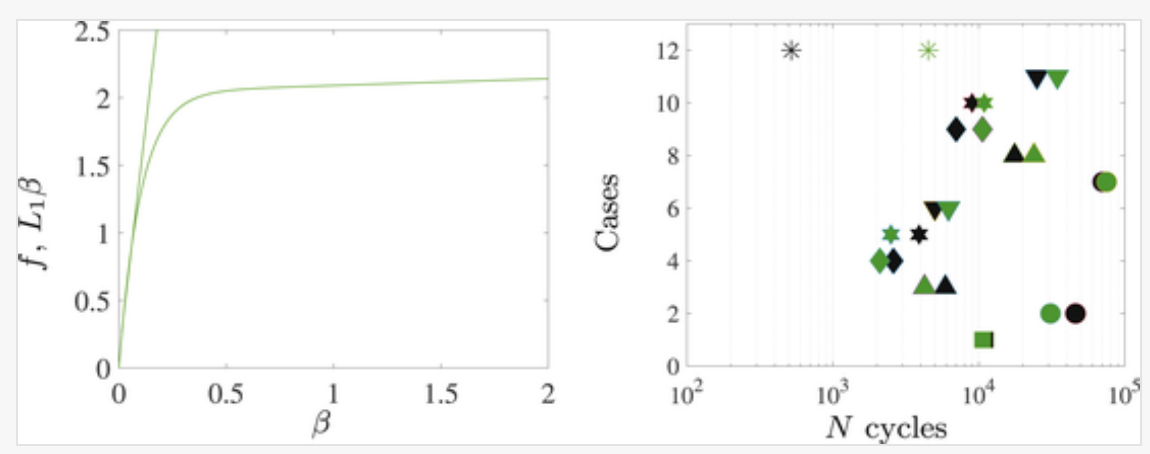


Functions $f$ and $L_{1} \beta$ (left). Fatigue lives (right). The loading cases $1-6$ are the tensional, torsional, elliptic, butterfly-type, squared, and rhombic as the maximum stress is $50 \%$ of the peak yield stress (tension) and ultimate shear strength. Cases $7-11$ are torsional, elliptic, butterfly-type, squared, and rhombic as the maximum stress is $30 \%$. Case 12 is the tensional as the maximum stress is $97 \%$. Black and green colors depict the observed mean values and predicted values, respectively. (For interpretation of the references to color in this figure legend, the reader is referred to the Web version of this article.)

The constitutive equations shown in Fig. 6 are regarded as uncoupled from fatigue, i.e. the system consisting of equations (13) and (14) is solved once the constitutive variables are known. This concept is motivated by our experimental observations showing that the fatigue damage represents practically the long-term formation of micro-cracks that typically covers most of (over 90\%) the total service life and that has not a marked influence on the macroscopic stresses and deformations, cf. Janssen et al. (2008); Hughes et al. (2017).

The model parameters are presented in Table 5. The initial value of $B$ was chosen to be 0.7 . The parameter $\sigma_{0}$ is the endurance limit (stress amplitude) for zero mean stress $(R=-1)$ while $a$ determines the corresponding slope of the Haigh-diagram, Ottosen et al. (2008). Since tensile specimens do not allow to investigate stress ratio $R=-1$, these parameters were extrapolated from the two uniaxial tensile tests: maximum stress $25 \%$ of the peak yield stress as $R=0.1$ and maximum stress $37.5 \%$ as $R=0.5(N \approx 60,000)$, see Fig. 7(right). It is assumed that the Haigh-diagram for the endurance limit is close to that used in the fitting. In the second phase, also a third uniaxial test with the maximum stress of $37.5 \%(R=0.1, N \approx 20,000)$ was applied to determine the HCFparameters $C, K$, and $L_{1}$ using the least squares fitting. In the third phase, the parameters $L_{2}$ and $\vartheta$ for LCF were extracted from the uniaxial test with the maximum stress of $90 \%(R=0.1)$, meanwhile $C, K$, and $L_{1}$ were slightly refined. Fourth, the parameter $c$ was defined based on the uniaxial test with the maximum stress of $90 \%$ and $R=0.5$. Then, the parameters $M$ and $Q$ were fitted to the torsion test with the maximum stress of $75 \%$ of the ultimate shear strength, $40 \mathrm{MPa}$. Finally, the last parameter $Q_{b}$ was fitted to data for the elliptic loading with the maximum stress of $50 \%$.

\section{alt-text: Table 5 \\ Table 5}

The table layout displayed in this section is not how it will appear in the final version. The representation below is solely purposed for providing corrections to the table. To preview the actual presentation of the table, please view the Proof.

Fatigue parameters.

\begin{tabular}{|c|c|c|c|c|c|c|c|c|c|c|c|c|}
\hline Parameter & $\begin{array}{l}\tau_{0}[\text { Instruction: } \\
\text { Isigma_0] }\end{array}$ & $a$ & $C$ & $K \cdot 10^{5}$ & $L_{1}$ & $L_{2}$ & $\vartheta$ & $\mathbf{M}$ & $Q$ & $Q_{b}$ & $c$ & B \\
\hline Unit ........... & $\mathrm{MPa}$ & & & & & & & & & $\mathrm{MPa}^{-1}$ & $\mathrm{MPa}^{-1}$ & \\
\hline Value .......... & 8.13 & 0.18 & 3.0 & 1.6 & 30 & 30 & 440 & 1.3 & 0.027 & 0.002 & 0.018 & 0.7 \\
\hline
\end{tabular}

The predicted vs observed fatigue lives are shown in Fig. 8. Considering the cases 1-11, the model captures the fatigue lives relatively accurately: the required safety factor is 1.5 . Under the heavy uniaxial loading case 12 , the model deviates significantly from the data point owing to the rapidly increasing ratcheting deformation leading to a brittle failure mechanism (not fatigue damage). The deformation behavior including ratcheting is examined in more detail below. 


\subsection{Uniaxial tension}

\subsubsection{Displacement-control}

One of the most important features of the model is its ability to predict the strain-rate dependence. The capability of the model in predicting this is demonstrated in Fig. 9. It can be observed that the model can predict the increasing peak yield stresses with increasing strain rates with reasonable accuracy. After reaching the peak yield stress, the tests showed brittle failure whereas the model, because it does not include brittle damage, produces significant softening at large strains, cf. e.g. Boyce et al. (1989); Holopainen (2013).

\section{alt-text: Fig. 9}

\section{Fig. 9}
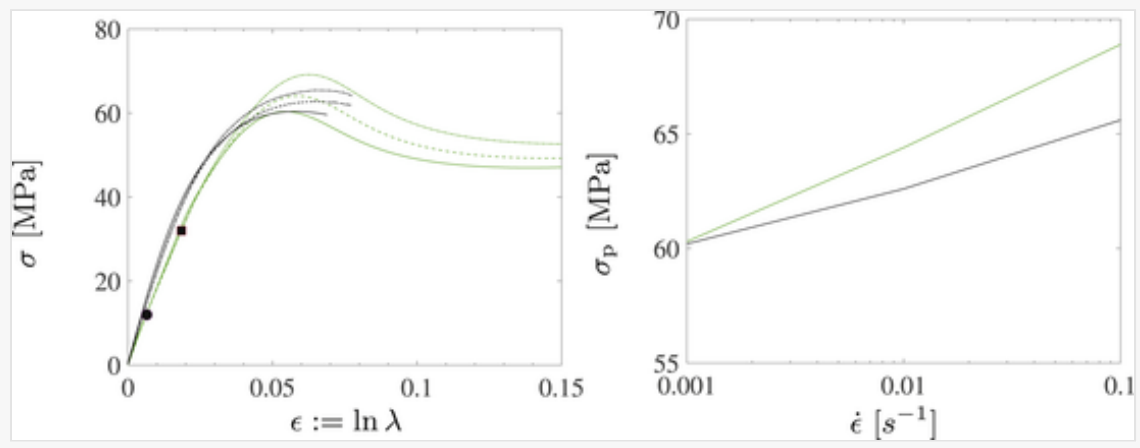

Cauchy stress-strain curves obtained from the uniaxial tensile tests under different strain rates of $0.001 s^{-1}$ (solid curve), $0.01 s^{-1}$ (dashed curve), and $0.1 s^{-1}$ (dashed-dotted curve) (left). The black and green curves represent the data and model responses. The - and symbols indicate the points when the viscoelastic and viscoplastic micro-mechanisms by the elements (2) (variable $\left.s^{(2)}\right)$ and (1) (variable $s^{(1)}$ ) shown in Fig. 6 start exhibiting notable effects. Peak yield stress vs strain rate (right). (For interpretation of the references to color in this figure legend, the reader is referred to the Web version of this article.)

\subsubsection{Load-control: creep-recovery test}

The ability to predict creep and recovery is also an important characteristic when evaluating viscous models. The tests and simulation concern stress levels under the peak yield stress, $60 \mathrm{MPa}$, suitable for long-term loadings in practise. ${ }^{5}$ Referring to Fig. 10 for a three step loading history, the predicted creep and recovery (nearly zero) are in good quantitative agreement with the experimental observations, because the difference in strains remains below 0.003 (15\% of the measured strain range). Both the model and data show a decreasing creep rate (primary creep) followed by a steady-state value (secondary creep), and the secondary creep reduces with increasing stress levels. A comparison to Fig. 9(left) (stress levels) reveals that the creep strain during the two first step is virtually viscoelastic, whereas plasticity governs the creep during the last step. 

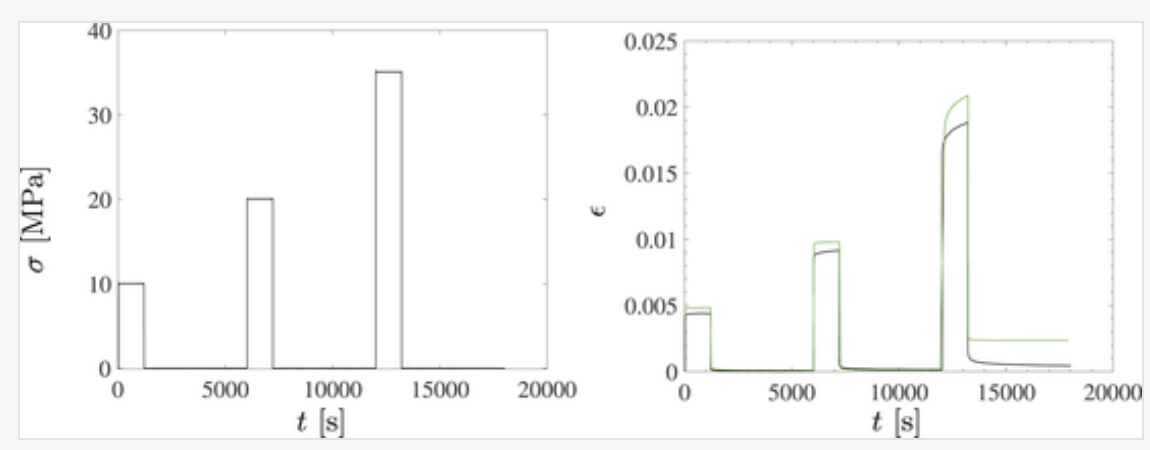

Loading for creep and recovery (left). Measured and predicted strains (right). The black and green curves implicate the test and model responses. The increasing and reducing stress rates were $60 \mathrm{MPa} / \mathrm{s}$. (For interpretation of the references to color in this figure legend, the reader is referred to the Web version of this article.)

\subsubsection{FE-simulation of load-controlled cyclic tests}

The model was also implemented in Abaqus Standard/Implicit, and a user material subroutine (UMAT) was compiled for this purpose. In this treatment, the algorithmic tangent stiffness (ATS) tensor is of major importance; it is discussed in e.g. Holopainen and Barriere (2018). The geometry of the tensile specimen is shown in Fig. 11. Owing to the symmetry of the geometry and boundary conditions, only a quarter of the specimen is modeled in the numerical analysis. The enlarged boundary is clamped and the axial cyclic pressure in the symmetry plane (middle of the web) is prescribed.

alt-text: Fig. 11

Fig. 11

Axial strain (logarithmic) distribution after 60 cycles as $\sigma=\sigma_{\min }, R=0.1, f=5 \mathrm{~Hz}$, and the maximum stress is $90 \%$ of the peak yield stress, $60 \mathrm{MPa}$.

Fig. 11 shows the axial strain distribution in the specimen right after the last unloading. ${ }^{6}$ The porosity distribution of the material in terms of the free volume was depicted in Fig. 1 showing that the free volume develops, as can be expected before significant ratcheting and necking, most evidently between the web and holder. It can be concluded that both deformation and porosity eventually develop mostly in the web of the test specimen, Holopainen (2014). Based on this observation, the following simulations are performed at a single material (integration) point in the middle of the specimen.

\subsubsection{Investigation of ratcheting}

The impact of the loading frequency, predicted by the model, is demonstrated in Fig. 12(left). The material shows hardening, i.e. stretching reduces with increasing loading frequency, cf. Boyce et al. (1995); Lu et al. 
(2016). Strain hardening is also evident from Fig. 9 as displacement-control is applied.

alt-text: Fig. 12

Fig. 12
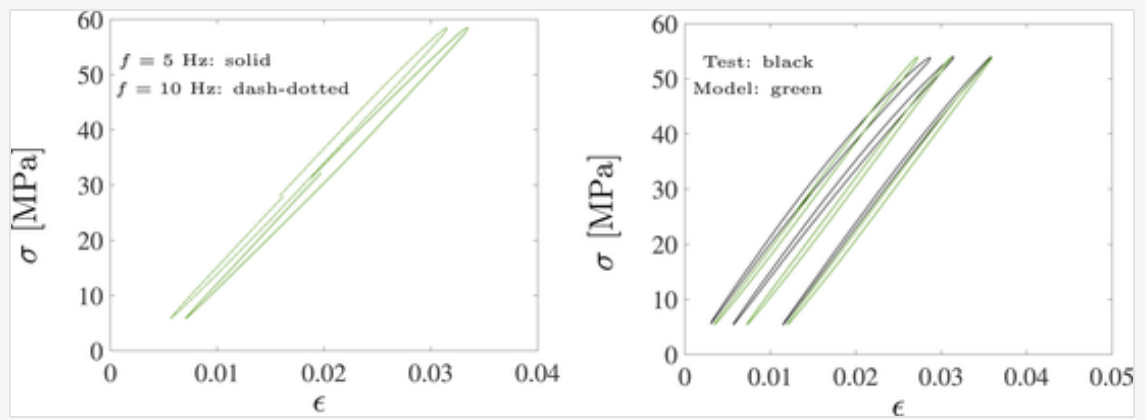

30th hysteresis loops by the model with $R=0.1$ and different loading frequencies (left). Although omitted for clarity, the loops with $f=5 \mathrm{~Hz}$ and $f=1 \mathrm{~Hz}$ almost overlap each other. 30th, 300th, and 3000th loop when $f=5 \mathrm{~Hz}$ (right).

Fig. 12(right) further shows selected hysteresis loops under the maximum stress close to the peak yield stress, $60 \mathrm{MPa}$. Hysteresis loops exhibit ratcheting (also termed cyclic creep), i.e. the mean strain grows as the applied load varies between its maximum and minimum values, cf. Jiang et al. (2015); Holopainen et al. (2017); Kang and Kan (2017). A comparison of Figs. 11 and 12(right) reveals also that the predicted strain values in the center of the specimen (by the finite-element simulation) and at a single material point are close to each other and agree well the experimental result.

To demonstrate the ratcheting behavior precisely, the ratcheting (mean) strain is defined by

$$
\varepsilon_{\mathrm{r}}:=\frac{1}{2}\left(\varepsilon_{\min }+\varepsilon_{\max }\right)
$$

wherein $\varepsilon_{\min }$ and $\varepsilon_{\max }$ mean the minimum strain and the maximum strain in each cycle. In the following, the observed rupture is illustrated by the marker $\mathbf{m}$, and the predictions end when full damage $(D=1)$ is achieved. Considering the highest load level in Fig. $13(R=0.1)$, the experimental response rapidly increases and intersects the model response after 100 cycles. The rapid increase in the experimentally observed ratcheting strain is due to a brittle failure mechanism (not fatigue damage), such as scission of chains, Vernerey et al. (2018) . The high loading also affects the temperature growth, resulting in slightly non-isothermal conditions and thus, softening of the material. At the lower stress levels, as shown in Fig. 13, the temperature increase remains low, and the proposed model captures the ratcheting strains and fatigue lives well.

alt-text: Fig. 13 


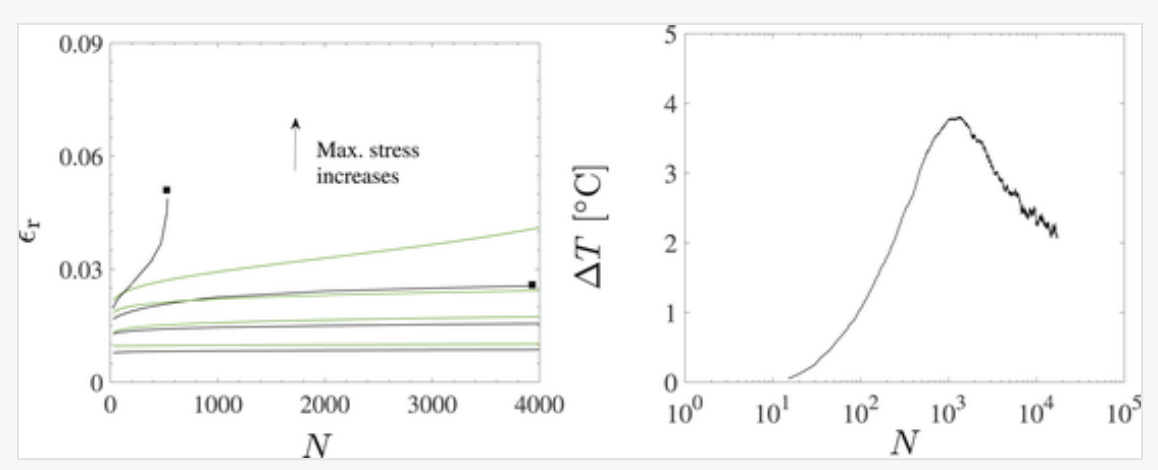

Ratcheting strain responses with $R=0.1$ and the frequency $f=5 \mathrm{~Hz}$. The maximum stresses investigated are $97 \%, 90 \%, 75 \%$, and $50 \%$ of the peak yield stress, $60 \mathrm{MPa}$ (left). The black and green colors imply the data and model results, and the $\mathrm{m}$ symbols refer to observed rupture: 525,3,940,5,580, and 10,590 cycles (predicted numbers of cycles are 4,140, 4,730, 5,450, and 10,610). The temperature rise during the loading as the maximum stress level is $90 \%$ (right). (For interpretation of the references to color in this figure legend, the reader is referred to the Web version of this article.)

Figs. 13 and 14 show that the impact of the loading level and stress ratio $R$ on the ratcheting strain and fatigue life is strong:[Instruction: Add a gap between the symbols and text.]

- Ratcheting strain $\varepsilon_{\mathrm{r}}$ increases with mean stress and amplitude;

- The higher $R$ and mean stress increase $\varepsilon_{\mathrm{r}}$ despite the lower stress amplitude, although

- the higher stress amplitude increases $\varepsilon_{\mathrm{r}}$ when the maximum stress is almost equal to the peak yield stress;

- fatigue life increases with the stress ratio $R$ (fixed maximum stress);

- fatigue life increases by the same percentage at both stress ratios as the maximum stress reduces (to about two-fold when the maximum stress reduces from $90 \%$ to $50 \%$ ).

alt-text: Fig. 14

\section{Fig. 14}

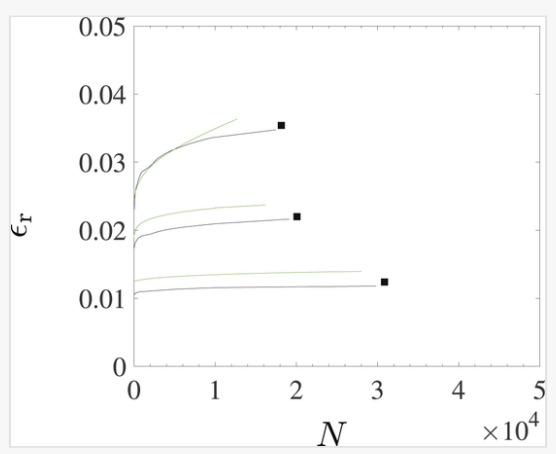

Ratcheting strain responses with $R=0.5$ and the frequency $f=5 \mathrm{~Hz}$. The maximum stresses investigated are $90 \%, 75 \%$, and $50 \%$ of the peak yield stress. The black and green colors imply the data and model results, respectively. The predictions end when the full damage $(D=1)$ has been reached, and the marker $\boldsymbol{a}$ refers to observed rupture. (For interpretation of the references to color in this figure legend, the reader is referred to the Web version of this article.)

Experimental responses right before rupture show also small but rapid increase in the ratcheting strains. This feature, as will be shown, is more evident under torsion and is a good indicator for the service lives.

\subsection{Torsion}




\subsubsection{Monotonic loadings}

The capability of the model in predicting torsion is demonstrated in Fig. 15(left). Under force control, the model response first increases and then attains its maximum value, whereas the experimental curve shows a strong decrease once the ultimate strength is reached. The observed reduction in stress results from material failure that ultimately leads to rupture at $\gamma=0.38$. As the displacement-control is applied, an initial increase of stress is followed by a notable reduction indicating strain softening, Fig. 15(right). The maximum or peak yield stress also increases with increasing deformation rate, which is line with the experiments, Boyce et al. (1995); Krairi and Doghri (2014); Lu et al. (2016).

alt-text: Fig. 15

\section{Fig. 15}

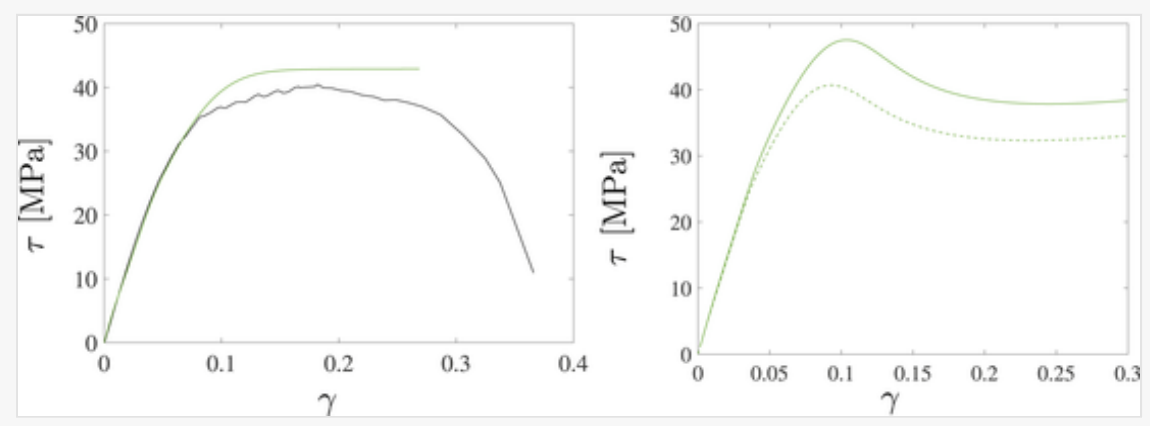

Torsion stress vs shear strain according to the force-controlled test (black) and model (green) (left). Predicted displacementcontrolled torsion for $\dot{\theta}=2^{\circ} / \mathrm{s}$ (solid curve) and $\dot{\theta}=0.1^{\circ} \mathrm{s}$ (dashed curve) (right). (For interpretation of the references to color in this figure legend, the reader is referred to the Web version of this article.)

\subsubsection{FE-simulation of load-controlled cyclic torsional tests}

The tests are simulated at a load level reflecting long-term service lives in practice $(50 \%$ of the ultimate shear strength). For the simulation, the rigid end of the test specimen shown in Fig. 2 has been replaced by a rigid attachment. Owing to the symmetry, only half of the specimen is modeled, and the symmetry plane is affected by a cyclic shear stress. The time step is limited in cyclic loadings, and a constant value of $0.01 \mathrm{~s}$ is used to extract the load spectrum sufficiently accurately. For simulating longer service lives, a specific time step algorithm is required, Haouala and Doghri (2015).

Fig. 16 shows the shear strain distribution in the torsion specimen immediately after the last unloading. Owing to the specimen's geometry based on the standard ASTM E2207 (Table 1), the shear strain distribution in the web is uniform. Therefore, the following calculations are performed in a single material point within this region. 
LE, Max. Principal

(Avg: $75 \%$ )
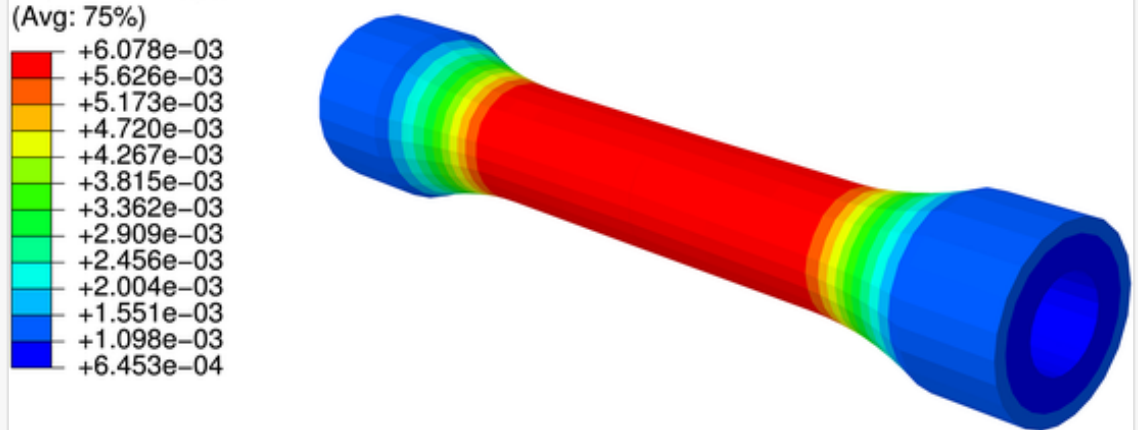

Shear strain (logarithmic) distribution after 50 cycles as $\tau=\tau_{\min }, R=0.1, f=1 \mathrm{~Hz}$, and the maximum shear stress is $50 \%$ of the ultimate shear strength, $40 \mathrm{MPa}$.

\subsubsection{Investigation of torsional ratcheting}

Considering first the maximum stress level $75 \%$ of the ultimate shear strength, the loops up to 10,000 cycles are close to the material response, see Fig. 17(left). As failure, such as chain breakage, starts to take a notable effect, the measured deformation increases, the material stiffness shows a reduction, and the area of the loops increases (a loss of mechanical energy or dissipation). The reduction in the material stiffness is most evident as the maximum stress of 50\% is applied, see Fig. 17(right). Because deformations are considered uncoupled from fatigue damage, the model is not capable of producing these characteristics, but reflects permanent chain deformation instead of chain breakage. However, as shown in Fig. 17(right), the reduction in the material stiffness and the loss of mechanical energy actually initiate when $90 \%$ of the fatigue life has been reached.

alt-text: Fig. 17

\section{Fig. 17}
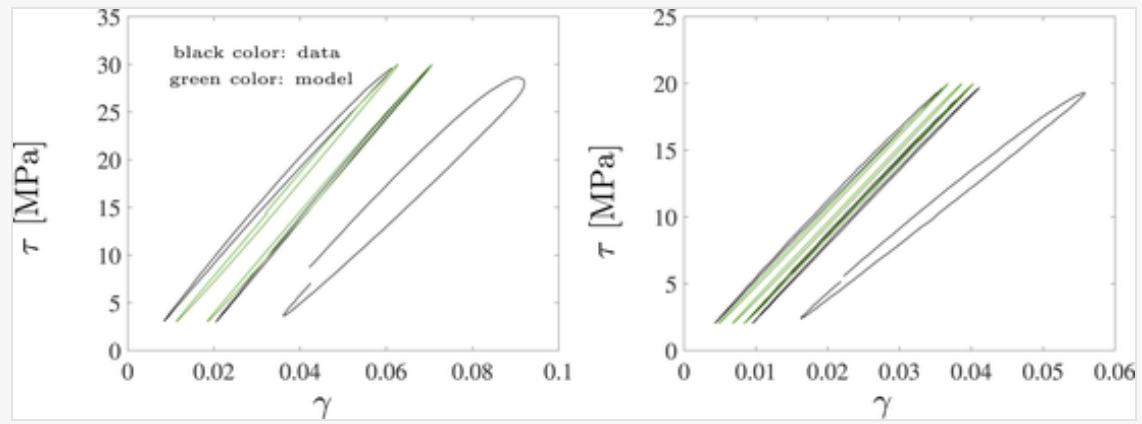

Torsional test and model responses for the maximum stress $75 \%$ of the ultimate shear strength, $40 \mathrm{MPa}$ (left). 30th, 10,000th, and 19,100 (right before rupture) test cycles, and 30th and 10,000th model cycles are shown. Test and model responses for $50 \%$ (right). 30th, 10,000th, and 40,000th cycles are shown. Also the measured 49,000th cycle (right before rupture) is shown.

Define the torsional ratcheting strain as

$$
\gamma_{\mathrm{r}}:=\frac{1}{2}\left(\gamma_{\min }+\gamma_{\max }\right)
$$

wherein $\gamma_{\min }$ and $\gamma_{\max }$ represent the minimum and maximum shear strains in each cycle. As with tension, the highest experimental response shows a rapid increase of ratcheting strain, which is caused by failure, such as 
scission of chains, Fig. 18(left). The high loading also increases temperature and thus, causes softening of the material. Owing to failure, the specimen collapsed at about 60 cycles, whereas the model predicts a steady increase in strain. Otherwise, the model well reproduces the measured ratcheting strain responses and fatigue lives.

\section{alt-text: Fig. 18}

\section{Fig. 18}
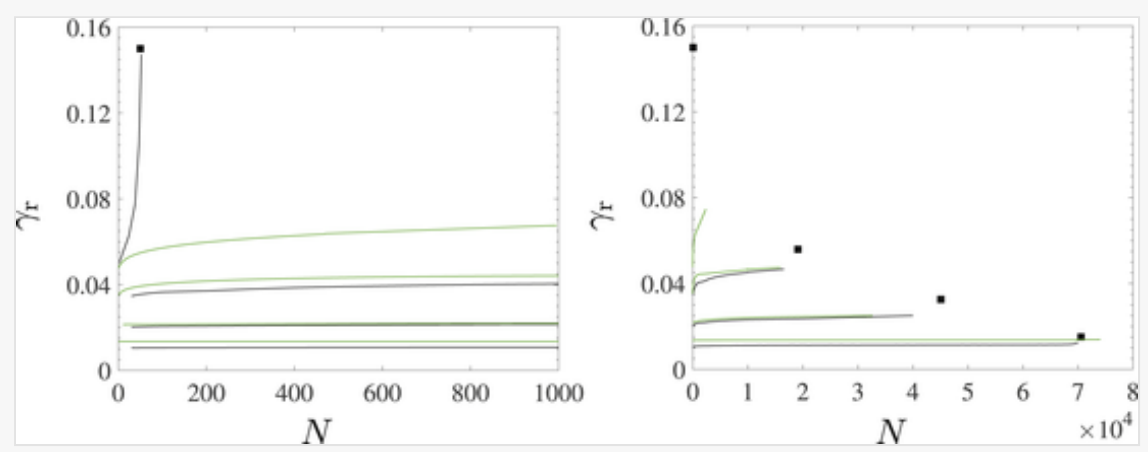

Initial (left) and whole (right) ratcheting strain responses with $R=0.1$ and the frequency $f=1 \mathrm{~Hz}$ for torsion. The values of maximum stresses investigated are $90 \%, 75 \%, 50 \%$, and $30 \%$ of the ultimate strength. The black and green colors indicate the experimental data and model results, respectively. Markers a refer to observed rupture. At the stress level $25 \%$, the fatigue life was about 580,000 cycles by both the test and model. (For interpretation of the references to color in this figure legend, the reader is referred to the Web version of this article.)

\subsection{Combined tension - torsion}

The multi-axial deformation behavior of PC is investigated under load-controlled cyclic loading conditions. The loading paths are illustrated in Fig. 4. To describe multi-axial loading in one dimension, the equivalent stress

$$
\sigma_{\mathrm{eqv}}:=\sqrt{\sigma^{2}+(\sqrt{3} \tau)^{2}}
$$

and equivalent strain

$$
\varepsilon_{\text {eqv }}:=\sqrt{\varepsilon^{2}+(\gamma / \sqrt{3})^{2}}
$$

are defined, cf. Lu et al. (2016); Shrivastava et al. (2012). Additionally, the ratcheting strain $\gamma_{\mathrm{r}}$ in (17) is replaced by the equivalent ratcheting strain $\gamma_{\mathrm{er}}=\gamma_{\mathrm{r}} / \sqrt{3}$.

\subsubsection{Influence of phase difference}

With the phase shift of zero, the hysteresis loops are narrow as depicted in Fig. 19. However, a relatively small phase shift in the shear stress already shows a different, elliptic shape of the loops, Fig. 20(right). The model seems to underestimate the shear strain at both load levels. However, as depicted in Fig. 21(left) and 22(left) for the equivalent hysteresis loops and ratcheting strains, respectively, the differences between the predicted and measured strain values can be considered small, and no conclusions can be drawn without investigating other loading cases. 


\section{Fig. 19}
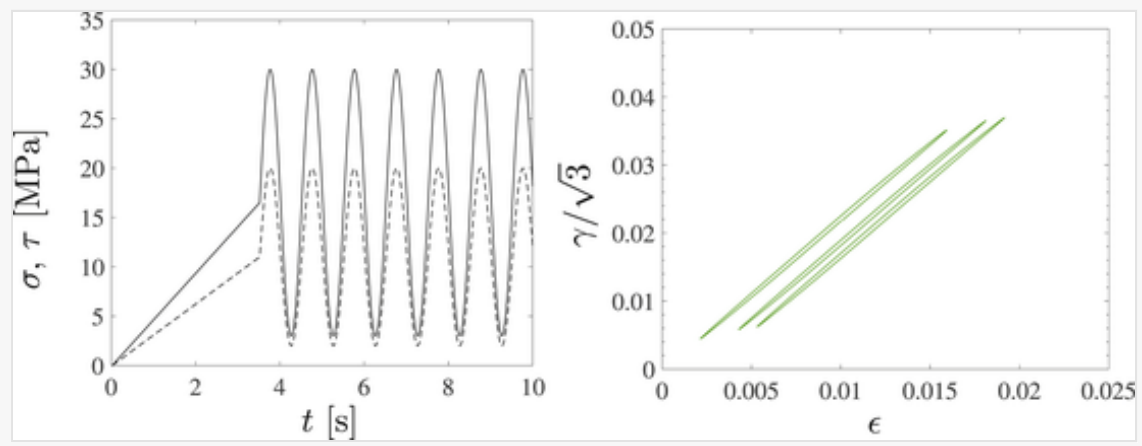

Loading path (cylindrical) without phase difference (left). Sinusoidal axial and shear stress signals are highlighted by the solid and dashed curves. Predicted axial strain vs shear strain (right). The 2nd, 1000th, and 6000th cycles are shown.

alt-text: Fig. 20

\section{Fig. 20}
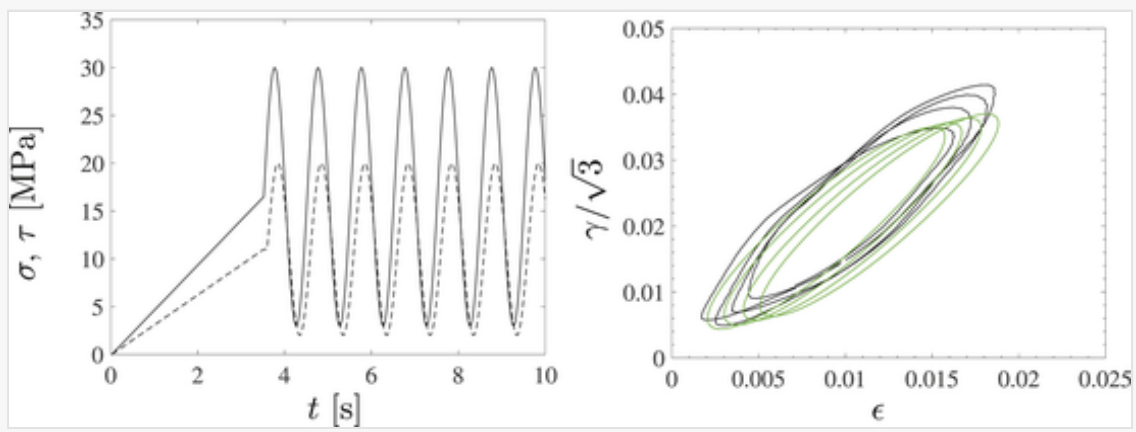

Loading path (elliptic) as the phase shift of shear stress is $\varphi=0.5$ (left). Axial and shear stresses are highlighted by the solid and dashed curves. Predicted (green) and measured (black) axial strain vs shear strain responses (right). The 2nd, 100th, 1000 th, and 6000th cycles are shown. (For interpretation of the references to color in this figure legend, the reader is referred to the Web version of this article.)

alt-text: Fig. 21

Fig. 21
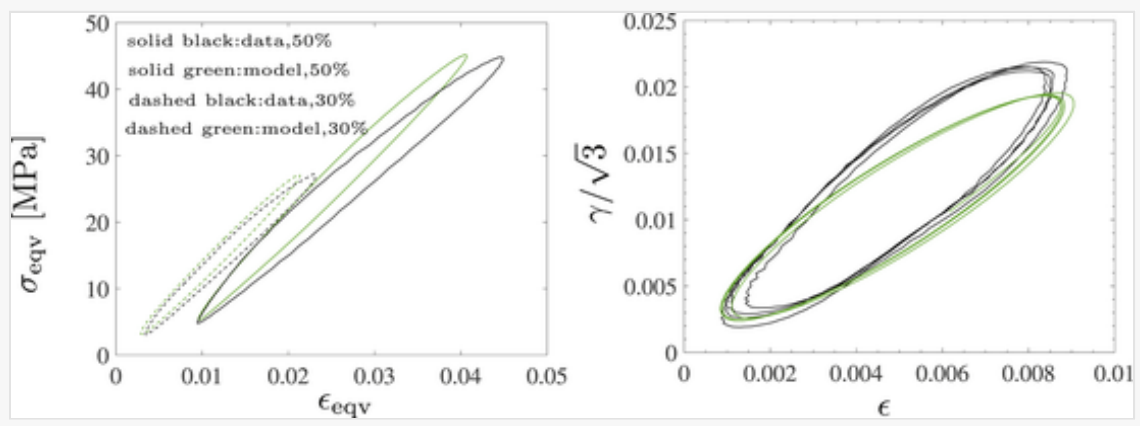

The 3000th loops for equivalent strain vs equivalent stress under the loading shown in Fig. 20 and when the maximum stress level is $30 \%$ of the peak yield stress (tension) and ultimate shear strength (left). Predicted (green) and measured (black) axial strain vs shear strain responses as the maximum stresses are 30\% (right). The 2 nd, 100th, 1000th, and 10,000th cycles are shown. (For interpretation of the references to color in this figure legend, the reader is referred to the Web version of this article.) 


\section{Fig. 22}
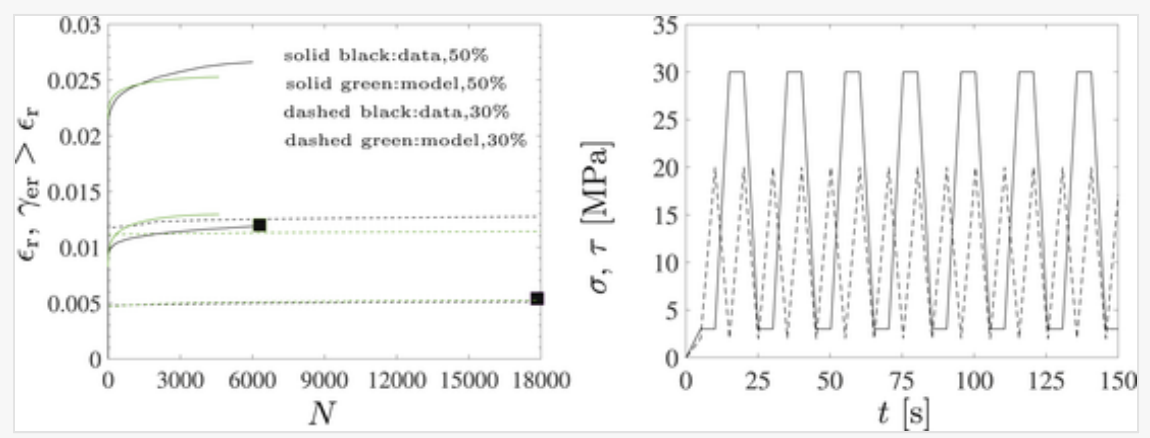

Predicted (green) and measured (black) axial and shear ratcheting strain responses for elliptic loading as the maximum stresses are $50 \%$ (solid curve) and 30\% (dashed curve) of their strengths (left). Of the same types of curves, the upper is the shear ratcheting. - symbols refer to observed rupture. Butterfly-type loading path (right). Axial and shear stresses are highlighted by the solid and dashed curves. (For interpretation of the references to color in this figure legend, the reader is referred to the Web version of this article.)

\subsubsection{Butterfly-type loading}

Fig. 22 shows the loading path for the first butterfly-type loading applied, and the hysteresis loops in terms of strains are shown in Fig. 23. As with linear and elliptic loadings, shear strain is considerably greater than the axial strain. A notable difference between the predicted strains and the data after $80 \%$ of the measured fatigue life is due to material degradation that is not considered in the model, i.e. the data show an increased deformation whereas the model starts to predict stabilized ratcheting. Otherwise, the model seems to be capable of predicting the deformation behavior, i.e. the shape of the loops as illustrated in Figs. 23-25(left).

alt-text: Fig. 23

\section{Fig. 23}
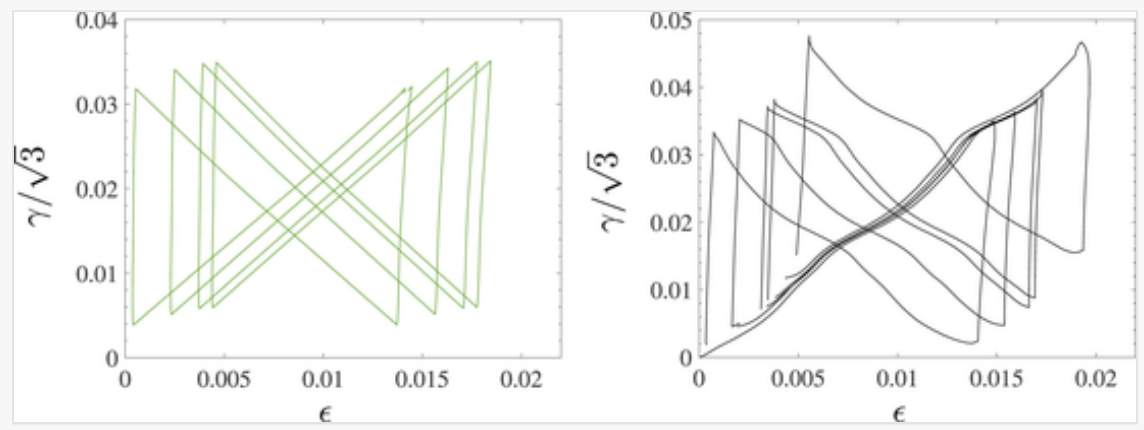

Predicted (left) and measured (right) axial strain vs shear strain responses under the stress responses shown in Fig. 22(right). The 2nd, 100th, 1000th, 2800th, and 3700th cycles are shown. Test specimen collapsed after the last cycle shown. 

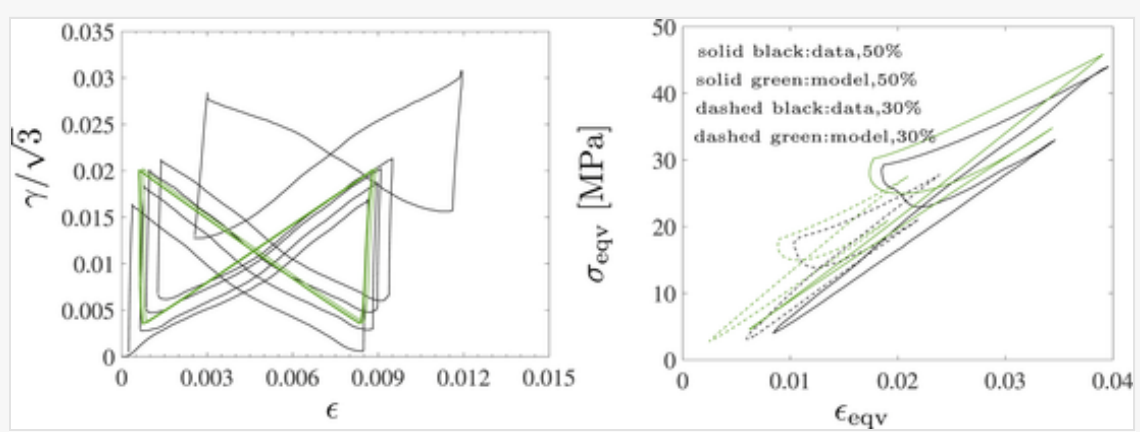

Predicted (green) and measured (black) axial strain vs shear strain responses as the axial and shear stresses are $30 \%$ of their strengths (left). The 2nd, 100th, 1000th (virtually overlapping by the model), and 5000th cycles are shown. Also, the 6940th cycle measured right prior to rupture is shown. Equivalent strain vs equivalent stress predicted and measured under the stress responses shown in Fig. 22 (2000th loop) and when the maximum stresses of 30\% are applied (3000th loop) (right). (For interpretation of the references to color in this figure legend, the reader is referred to the Web version of this article.)

alt-text: Fig. 25

\section{Fig. 25}

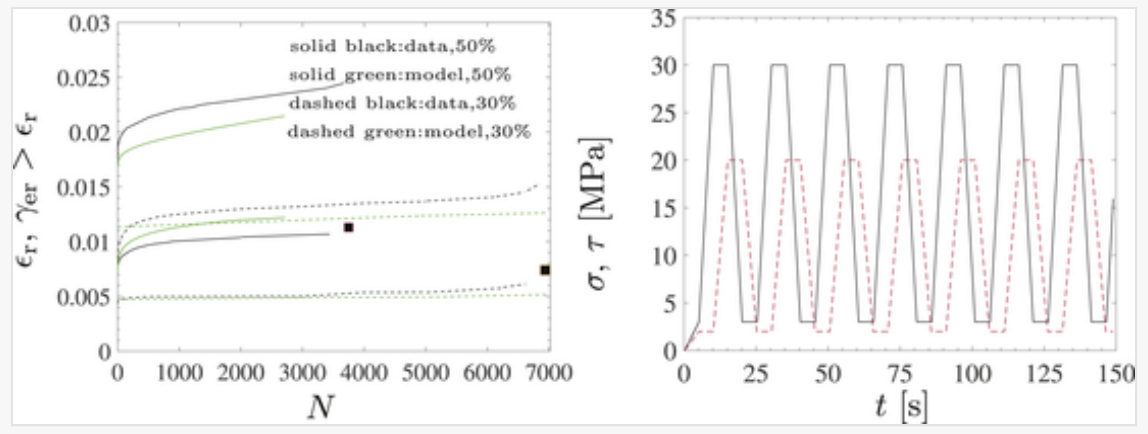

Predicted and measured ratcheting strain responses for butterfly-type loading as the maximum stress levels applied are 50\% and $30 \%$ (left). Markers - refer to observed rupture (95\% of the fatigue life). Square-type loading path (right). Axial and shear stresses are marked by the solid and dashed curve.

\subsubsection{Square-type loading}

The square-type loading is illustrated in Fig. 25. The measured and predicted strain responses shown in Fig. 26 (left) reveals that the model underestimates the maximum of shear strain and overestimates uniaxial strain. Under the lower stress level of $30 \%$, however, the difference between the model result and data is small, i.e. it is the viscoplastic response that is too stiff in shear and too soft in tension. Considering the latter, the pre-peak slope of the axial stress-strain response is slightly too low and could be increased (parameters $h_{0}$ and $b$ ), see Fig. 9(left). The model's rigidity in shear is likely to be related to the applied eight-chain model that has been found to be quite rigid under high shear loads, Wu and Van der Giessen (1993); Boyce et al. (1995). 

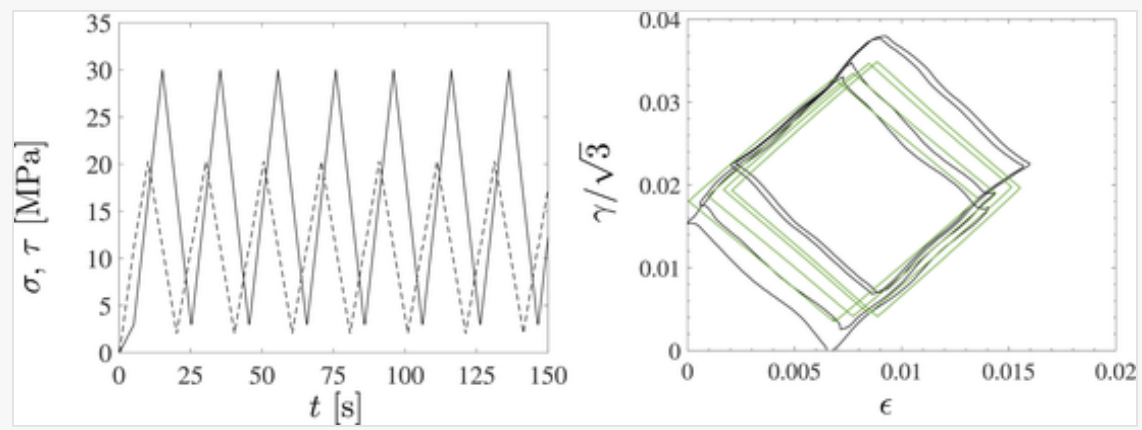

Rhombic loading path (left). Axial and shear stresses are highlighted by the solid and dashed curve, respectively. Predicted (green) and measured (black) axial strain vs shear strain responses (right). The 2nd, 100th, 1000th, and 3000th cycles are shown. (For interpretation of the references to color in this figure legend, the reader is referred to the Web version of this article.)

alt-text: Fig. 29

\section{Fig. 29}
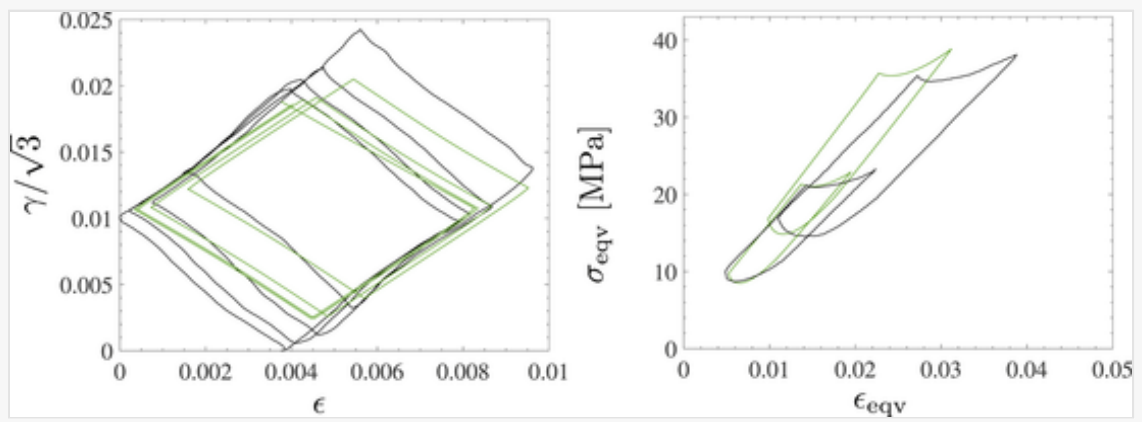

Predicted (green) and measured (black) axial strain vs shear strain responses as the maximum stress level is $30 \%$ (left). The 2nd, 100th, 1000th, and 24,000th cycles are shown. Predicted and measured 3000th loop for equivalent strain vs equivalent stress (right). (For interpretation of the references to color in this figure legend, the reader is referred to the Web version of this article.)

alt-text: Fig. 30

\section{Fig. 30}

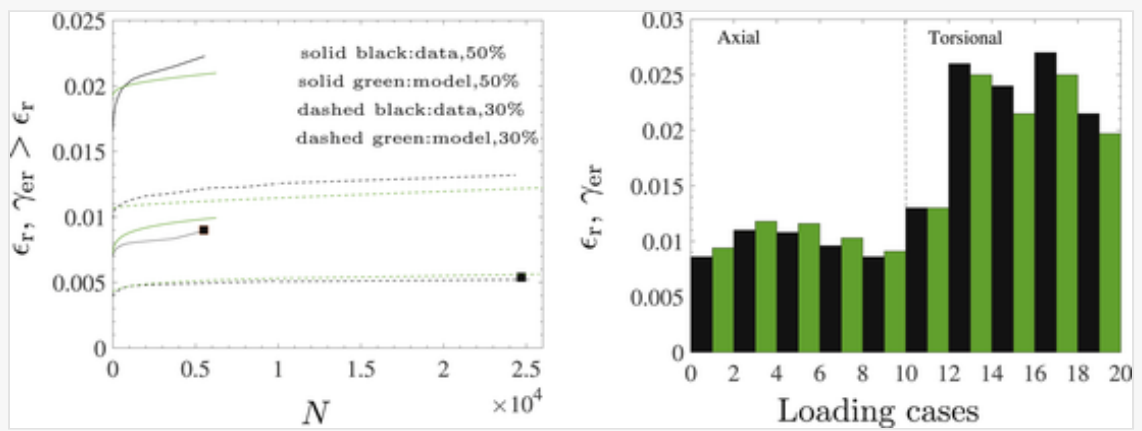

Predicted (green) and measured (black) axial and shear ratcheting strain responses for the rhombic loading (left). a symbol refers to observed rupture. At the lowest level, rupture occurred on 24,580 cycles. Predicted and measured (black) axial (cases 1-10) and torsional (cases 11-20) ratcheting strains at 2000 cycles (right). The loading cases 2-10 and 12-20 are the elliptic, butterfly-type, squared, and rhombic. Cases 1 and 11 are pure tension and torsion. (For interpretation of the references to color in this figure legend, the reader is referred to the Web version of this article.) 


\subsubsection{Comparison of the loading paths}

The same maximum stress levels (50\% of the strengths) and the same stress ratio $R=0.1$ have been used. The results are summarized in Fig. 30 at 2000 cycles (before observed rupture and reduction of shear stiffness). It should be noted that different loading frequencies of $f=0.05, f=1$, and $f=5 \mathrm{~Hz}$ are applied under the combined tension/torsion (butterfly, squared, rhombic), elliptic loading/pure torsion, and tension, respectively. However, as shown in Fig. 12, the impact of the loading frequencies less than $5 \mathrm{~Hz}$ on ratcheting strain is small. It can be observed that torsional ratcheting is emphasized in all cases. Differences between axial ratcheting strains are small, whereas the superimposed cyclic tension apparently increases the shear strain. This feature is due to the high axial tension relative to the torque and also appears under lower loadings, as shown by comparing the results in Figs. 13, 18 and 22(left), 25(left), 27(right), and 30. The rhombic loading shows the minimum tensile ratcheting by both the data and model, whereas the squared-type loading underscores torsional ratcheting.

It can also be observed that the model underestimates the shear ratcheting. The model's rigidity in shear is partially due to too stiff elastic response, i.e. the value of the shear modulus is too high (low Poisson's ratio), see Section 2.3. The exaggerated stiffness before rupture would further be reduced by considering damage evolution, see canonical [ $1-D$ ] effective stress concepts Junior et al. (2011); Wang et al. (2016). In overall, the model's rigidity in shear seems to be related to the applied eight-chain model, which model is quite rigid under shear loads, Wu and Van der Giessen (1993); Boyce et al. (1995). In conclusion, as has been shown in Fig. 30 (right), the differences between the predicted and measured deformations are rather small when the number of cycles is less than $90 \%$ of the fatigue life (before brittle material degradation).

\section{Concluding remarks and further research avenues}

Long-term deformation behavior of polymers under isothermal loadings was investigated. Based on a large test program, the effects of different loading modes, creep load conditions, mean stress, stress amplitude, and loading rate on a common PC polymer were studied. A viscoelastic-viscoplastic constitutive model introduced in Barriere et al. (2019) for solid polymers was used in the simulations. The model requires a reduced number of material parameters and it is easy to implement in finite-element packages. In addition, the model was supplemented with fatigue damage. It was shown that the results of the model are close to the experimental results, and thus the model could be a useful tool for simulating costly tests and in the product development. The following conclusions are drawn based on both the experiments and simulations (below the peak yield stress and before significant softening):

- The material shows an apparent strain hardening with increasing loading frequencies;

- Ratcheting strain depends strongly on the applied stress ratio $R$, and a higher $R$ and mean stress increase ratcheting, despite the lowered stress amplitude, although

- an increase in stress amplitude increases ratcheting when the maximum stress is near the peak yield stress;

- Superimposed cyclic tension noticeably increases torsional ratcheting, while

- variation in ratcheting strains between the different multi-axial loading cases is low;

- fatigue life increases with the stress ratio $R$ (fixed maximum stress) despite increased ratcheting;

- fatigue life increases by the same percentage at different stress ratios $R$ when the maximum stress reduces. 
The proposed approach could be developed further: the impact of temperature rice on energy dissipation, the influence of strain history (plasticity) on fatigue damage, and the reduction of shear stiffness due to damage are important research topics. In addition, microstructural changes control fatigue and them need to be investigated further.

\section{Declaration of competing interest}

The authors declare that they have no known competing financial interests or personal relationships that could have appeared to influence the work reported in this paper.

\section{Acknowledgements}

The authors acknowledge the technician Ly Phanousith from FEMTO-ST institute for conducting the experiments. This work has been supported by the EIPHI Graduate School (contract ANR-17-EURE-0002).

\section{Appendix Numerical solution method}

The general system of evolution equations for the finite element method is given in (Barriere et al., 2019, Appendix, B). The reduced system for uniaxial tension is also given therein. The evolution equations for modeling the force-controlled combined tension-torsion tests are defined below. To define the intermediate placement uniquely, the following restrictions are made:[Instruction: Add a gap between the symbols and text]

- The elastic deformation gradient $\boldsymbol{F}^{\mathrm{e}}$ is considered symmetric, i.e. $\boldsymbol{v}^{\mathrm{e}}$ in (3) is known through the constitutive law (elastic spin and viscoelastic-plastic rotation are non-zero), cf. Holopainen and Barriere (2018);

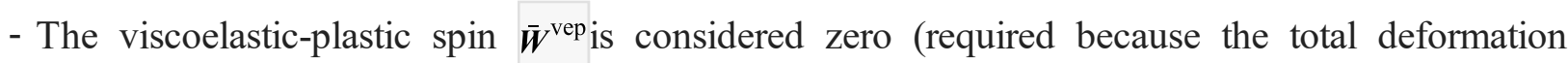
gradient under force control is unknown);

- The viscoelastic spin $\omega^{\text {ve }}$ is chosen to vanish to uniquely define also the relationship between the viscoelastic and plastic rotations.

The second restriction is based on the investigation showing that $\overline{\boldsymbol{W}}^{\text {vep }}$ is small and typically lower than elastic elongations, which are already very low in glassy polymers, Anand and Ames (2006). The variables to be solved are in their vectorized form as $\boldsymbol{Y}:=\left[\boldsymbol{F}^{\mathrm{vep}} \overline{\boldsymbol{C}}^{\mathrm{ve}} s^{(1)} \mu_{1} \phi\right]$, [Instruction: must be Ivarphi, not $\mid$ phi] where $\overline{\boldsymbol{C}}^{\mathrm{ve}}$ represents viscoelastic deformation and $s^{(1)}, \mu_{1}$, and $\phi$ [Instruction: must be Ivarphi, not Iphi]are internal variables defined in Fig. 6, cf. Barriere et al. (2019). The component matrix of $\boldsymbol{F}^{\text {vep }}$ is given in (8), i.e. solely the components $F_{11}^{\text {vep }}$ and $F_{21}^{\text {vep }}$ are unknown. Noting volume-preserving viscoelastic deformation,

$$
\bar{C}_{22}^{\mathrm{ve}}=\bar{C}_{33}^{\mathrm{ve}}=1 / \sqrt{\bar{C}_{11}^{\mathrm{ve}}}
$$

holds, and owing to the symmetry, $\bar{C}_{12}^{\mathrm{ve}}=\bar{C}_{21}^{\mathrm{ve}}$. In total, seven variables must be solved from the system[Instruction: the equal signs must be in line][Instruction: in R7, use Ivarphi instead of \phi] 


$$
\begin{array}{cc}
R_{1}: & =F_{11}^{\mathrm{vep}}-F_{\mathrm{n}, 11}^{\mathrm{vep}}-\Delta t\left(\bar{D}_{11}^{\mathrm{vep}} F_{11}^{\mathrm{vep}}+\bar{D}_{12}^{\mathrm{vep}} F_{21}^{\mathrm{vep}}\right), \\
R_{2}: & =F_{21}^{\mathrm{vep}}-F_{\mathrm{n}, 21}^{\mathrm{vep}}-\Delta t\left(\bar{D}_{21}^{\mathrm{vep}} F_{11}^{\mathrm{vep}}+\bar{D}_{22}^{\mathrm{vep}} F_{21}^{\mathrm{vep}}\right), \\
R_{3}: & =\bar{C}_{11}^{\mathrm{ve}}-\bar{C}_{\mathrm{n}, 11}^{\mathrm{ve}}-2 \Delta t\left(\bar{D}_{11}^{\mathrm{ve}} \bar{C}_{11}^{\mathrm{ve}}+\bar{D}_{12}^{\mathrm{ve}} \bar{C}_{21}^{\mathrm{ve}}\right), \\
R_{4}:=\bar{C}_{12}^{\mathrm{ve}}-\bar{C}_{\mathrm{n}, 12}^{\mathrm{ve}}-\Delta t\left(\bar{D}_{11}^{\mathrm{ve}} \bar{C}_{12}^{\mathrm{ve}}+\bar{D}_{12}^{\mathrm{ve}} \bar{C}_{22}^{\mathrm{ve}}+\bar{C}_{11}^{\mathrm{ve}} \bar{D}_{12}^{\mathrm{ve}}+\bar{C}_{12}^{\mathrm{ve}} \bar{D}_{22}^{\mathrm{ve}}\right), \\
R_{5}: & =\left(s^{(1)}-s_{\mathrm{n}}^{(1)}-\Delta t \dot{s}^{(1)}\right) / s_{0}, \\
R_{6}: & =\left(\mu_{1}-\mu_{1, \mathrm{n}}-\Delta t \dot{\mu}_{1}\right) / \mu_{1}^{0}, \\
R_{7}: & =\phi-\phi_{n}-\Delta t \dot{\phi},
\end{array}
$$

where the updated state is defined at the time instant $t=t_{n}+\Delta t$ ( $\Delta t$ is a time increment), and $n$ refers to the known state at $t_{n}$. The solution of system (A.2) is $\boldsymbol{Y}=\boldsymbol{Y}_{\mathrm{n}}+\Delta \boldsymbol{Y}$ and it is obtained by the Newton-Raphson method, where

$$
\Delta \boldsymbol{Y}=-\boldsymbol{J}^{-1} \boldsymbol{R}, \boldsymbol{J}=\frac{\partial \boldsymbol{R}}{\partial \boldsymbol{Y}}(\text { Jacobian}), \boldsymbol{R}=\left[R_{1} R_{2} R_{3} R_{4} R_{5} R_{6} R_{7}\right]
$$

Once $\boldsymbol{F}^{\text {vep }}$ is solved, the deformation gradient $\boldsymbol{F}$ is available from the decomposition (2), because $\boldsymbol{F}^{\mathrm{e}}$ is known from the constitutive description ( $\boldsymbol{R}^{\mathrm{e}}$ is unity).

\section{References}

(i) The corrections made in this section will be reviewed and approved by a journal production editor. The newly added/removed references and its citations will be reordered and rearranged by the production team.

Anand, L., Ames, N.M., 2006. On modeling the micro-indentation response of an amorphous polymer. Int. J. Plast. 22, 1123-1170.

ASTM D638, 2003. In: Designation: D 638 - 03. Standard Test Method for Tensile Properties of Plastics, vols. 1-15. ASTM International.

ASTM E2207, 2002. In: Standard Practice for Strain-Controlled Axial-Torsional Fatigue Testing with Thin- Walled Tubular Specimens, vols. 1-8. ASTM International.

Avanzini, A., 2008. Mechanical characterization and finite element modelling of cyclic stress-strain behaviour of ultra high molecular weight polyethylene. Mater. Des. 29, 330-343.

Barriere, T., Cheng, G., Holopainen, S., 2018. Modeling, simulation, and experimentation of fatigue behavior in amorphous solids. Key Eng. Mater. 774, 210-216.

Barriere, T., Gabrion, X., Holopainen, S., 2019. A compact constitutive model to describe the viscoelastic-plastic behaviour of glassy polymers: comparison with monotonic and cyclic experiments and state-of-the-art models. Int. J. Plast. 122, 31-48.

Beesley, R., Chen, H., Hughes, M., 2017. A novel simulation for the design of a low cycle fatigue experimental testing programme. Comput. Struct. 178, 105-118. 
Boyce, M.C., Arruda, E.M., Jayachandran, R., 1995. The large strain compression, tension, and simple shear of polycarbonate. Polym. Eng. Sci. 34, 716-725.

Boyce, M.C., Weber, G.G., Parks, D.M., 1989. On the kinematics of finite strain plasticity. J. Mech. Phys. Solid. 37, 647-665.

Chen, G., Liang, H.Q., Wang, L., Mei, Y.H., Chen, X., 2015. Multiaxial ratcheting-fatigue interaction on acrylonitrile-butadiene-styrene terpolymer. Polym. Eng. Sci. 55, 664-671.

Chen, K., Kang, G., Yu, C., Jiang, H., 2019. Effect of crystalline content on ratchetting of ultra-high molecular weight polyethylene polymers: experimental investigation and constitutive model. Mech. Mater. 113[Instruction: should be 133], 37-54.

Dreistadt, C., Bonnet, A.S., Chevrier, P., Lipinski, P., 2009. Experimental study of the polycarbonate behaviour during complex loadings and comparison with the Boyce, Parks and Argon model predictions. Mater. Des. 30, 3126-3140.

Haouala, S., Doghri, I., 2015. Modeling and algorithms for two-scale time homogenization of viscoelastic-viscoplastic solids under large numbers of cycles. Int. J. Plast. 70, 98-125.

Haward, R.N., Thackray, G., 1968. The use of a mathematical model to describe isothermal stress-strain curves in glassy thermoplastics. Proc. Roy. Soc. A 302, 453-472.

Holopainen, S., 2013. Modeling of the mechanical behavior of amorphous glassy polymers under variable loadings and comparison with state-of-the-art model predictions. Mech. Mater. 66, 35-58.

Holopainen, S., 2014. Influence of damage on inhomogeneous deformation behavior of amorphous glassy polymers. Modeling and algorithmic implementation in a finite element setting. Eng. Fract. Mech. 117, $28-50$.

Holopainen, S., Barriere, T., 2018. Modeling of mechanical behavior of amorphous solids undergoing fatigue loadings, with application to polymers. Comput. Struct. 199, 57-73.

Holopainen, S., Barriere, T., Cheng, G., Kouhia, R., 2017. Continuum approach for modeling fatigue in amorphous glassy polymers. applications to the investigation of damage-ratcheting interaction in polycarbonate. Int. J. Plast. 91, 109-133.

Hughes, J.M., Lugo, M., Bouvard, J.L., McIntyre, T., Horstemeyer, M.F., 2017. Cyclic behavior and modeling of small fatigue cracks of a polycarbonate polymer. Int. J. Fatig. 99, 78-86.

James, M.N., Lu, Y., Christopher, C.J., Patterson, E.A., 2013. Crack path support for deformation mechanisms in fatigue of polycarbonate. Eng. Fract. Mech. 108, 89-97.

Janssen, R.P.M., Kanter, D.K., Govaert, L.E., Meijer, H.E.H., 2008. Fatigue life predictions for glassy polymers: a constitutive approach. Macromolecules 41, 2520-2530.

Jiang, C.K., Jiang, H., Zhang, J.W., Kang, G.Z., 2015. A viscoelasticplastic constitutive model for uniaxial ratcheting behaviors of polycarbonate. Polym. Eng. Sci. 55, 2559-2565.

Junior, M.V., de Souza Neto, E.A., Munoz-Rojas, P.A., 2011. Advanced Computational Materials Modeling: from Classical to Multi-Scale Techniques. John Wiley \& Sons. 
Kang, G., Kan, Q., 2017. Cyclic Plasticity of Engineering Materials: Experiments and Models. John Wiley \& Sons, Chichester.

Kanters, M.J.W., Kurokawa, T., Govaert, L.E., 2016. Competition between plasticity-controlled and crack-growth controlled failure in static and cyclic fatigue of thermoplastic polymer systems. Polym. Test. $50,101-110$.

Kim, G.H., Lu, H., 2008. Accelerated fatigue life testing of polycarbonate at low frequency under isothermal condition. Polym. Test. 27, 114-121.

Kirkinis, E., Ogden, R.W., 2002. On extension and torsion of a compressible elastic circular cylinder. Math. Mech. Solid 7, 373-392.

Krairi, A., Doghri, I., 2014. A thermodynamically-based constitutive model for thermoplastic polymers coupling viscoelasticity, viscoplasticity and ductile damage. Int. J. Plast. 60, 163-181.

Krairi, A., Doghri, I., Schalnat, J., Robert, G., Van Paepegem, W., 2019. Thermo-mechanical coupling of a viscoelastic-viscoplastic model for thermoplastic polymers: thermodynamical derivation and experimental assessment. Int. J. Plast. 115, 154-177.

Krenk, S., Tidemann, L., 2017. A compact cyclic plasticity model with parameter evolution. Mech. Mater. $113,57-68$.

Lu, F., Kang, G., Zhu, Y., Xi, C., Jiang, H., 2016. Experimental observation on multiaxial ratchetting of polycarbonate polymer at room temperature. Polym. Test. 50, 135-144.

Ottosen, N., Stenström, R., Ristinmaa, M., 2008. Continuum approach to highcycle[Instruction: should be high-cycle] fatigue modeling. Int. J. Fatig. 30, 996-1006.

Qi, Z., Hu, N., Li, G., Zeng, D., Su, X., 2019. Constitutive modeling for the elastic-viscoplastic behavior of high density polyethylene under cyclic loading. Int. J. Plast. 113, 125-144.

Ravi Chandran, K.S., 2016. Mechanical fatigue of polymers: a new approach to characterize the S-N behavior on the basis of macroscopic crack growth mechanism. Polymer 91, 222-238.

Shojaei, A.K., Volgers, P., 2018. A coupled hyperelastic-plastic-continuum damage model for studying cyclic behavior of unfilled engineering polymers. Int. J. Fatig. 107, 33-39.

Shrivastava, S., Ghosh, C., Jonas, J., 2012. A comparison of the von Mises and Hencky equivalent strains for use in simple shear experiments. Philos. Mag. 92 (7), 779-786.

Vernerey, F.J., Brighenti, R., Long, R., Shen, T., 2018. Statistical damage mechanics of polymer networks. Macromolecules 51, 6609-6622.

Wang, J., Xu, Y., Zhang, W., Moumni, Z., 2016. A damage-based elasticviscoplastic constitutive model for amorphous glassy polycarbonate polymers. Mater. Des. 97, 519-531.

Wright, W.J., Dauskardt, R.H., Nix, W.D., 2003. The prospects for mechanical ratcheting of bulk metallic glasses. Mater. Res. Soc. MM7.5 806. 
Wu, P.D., Van der Giessen, E., 1993. On improved network models for rubber elasticity and their applications to orientation hardening in glassy polymers. J. Mech. Phys. Solid. 41, 427-456.

Xi, C., Kang, G., Lu, F., Zhang, J., Jiang, H., 2015. An experimental study on uniaxial ratcheting of polycarbonate polymers with different molecular weights. Mater. Des. 67, 644-648.

Yu, C., Kang, G., Chen, K., Lu, F., 2017. A thermo-mechanically coupled nonlinear viscoelasticviscoplastic[Instruction: should be viscoelastic-viscoplastic] cyclic constitutive model for polymeric materials. Mech. Mater. 105, 1-15.

Zhu, Y., Kang, G., Yu, C., 2017. A finite cyclic elasto-plastic constitutive model to improve the description of cyclic stress-strain hysteresis loops. Int. J. Plast. 95, 191-215.

\section{Footnotes}

\section{Text Footnotes}

[1] The highest stress before softening (displacement-control), cf. Dreistadt et al. (2009).

[2] The measured stress is actually the first Piola Kirchoff stress; the Kirchhoff stress applied in the model is $\tau:=\sigma \lambda$, where $\lambda=1+\varepsilon$ is the axial stretch.

[3] The polar decomposition of $\boldsymbol{F}^{\text {vep }}$ is not applied, because this deformation is path-dependent when the relationship between viscoplastic rotations and stretching is difficult to define.

[4] It can be shown that the endurance surface in a uniaxial loading reduces to the form $\sigma_{\mathrm{a}}+g_{\mathrm{I}}-\sigma_{0}=0$, cf. (Ottosen et al., 2008, eq. (12)).

\section{Highlights}

- Deformation behavior of polymers under uniaxial and multi-axial quasi-static loadings.

- Large test program consisting of long-term tension, torsion, and their combinations.

- Effects of loading modes and rates, creep load conditions, mean stress, and amplitude.

- Possibility of replacing costly tests with modeling. Based on the both:

- Hardening with increasing frequencies and superimposed tension increases torsional ratcheting.

\section{Queries and Answers}

Query: Your article is registered as a regular item and is being processed for inclusion in a regular issue of the journal. If this is NOT correct and your article belongs to a Special Issue/Collection please contact s.sadhasivan@elsevier.com immediately prior to returning your corrections.

Answer: Yes 
Query: Please confirm that given names and surnames have been identified correctly and are presented in the desired order and please carefully verify the spelling of all authors' names.

Answer: Yes

Query: Please confirm that the provided email "sami.holopainen@tuni.fi” is the correct address for official communication, else provide an alternate e-mail address to replace the existing one, because private e-mail addresses should not be used in articles as the address for communication.

Answer: Correct

Query: Have we correctly interpreted the following funding source(s) you cited in your article: EIPHI Graduate School?

Answer: Yes

Query: Please provide the volume number or issue number or page range or article number for the bibliography in Ref(s). Wright et al., 2003.

Answer: Volume is 806. 